LMU-TPW 1-01

LPT-ORSAY 01-22

UWThPh-2001-10

\title{
Field Theory on the $q$-deformed Fuzzy Sphere II: Quantization
}

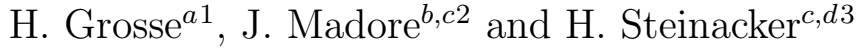 \\ ${ }^{a}$ Institut for Theoretical Physics, University of Vienna, \\ Boltzmanngasse 5, A-1090 Vienna, Austria \\ ${ }^{b}$ Max-Planck-Institut für Physik \\ Föhringer Ring 6, D-80805 München \\ ${ }^{c}$ Laboratoire de Physique Théorique et Hautes Energies \\ Université de Paris-Sud, Bâtiment 211, F-91405 Orsay \\ ${ }^{d}$ Sektion Physik der Ludwig-Maximilians-Universität München \\ Theresienstr. 37, D-80333 München
}

\begin{abstract}
We study the second quantization of field theory on the $q$-deformed fuzzy sphere for $q \in$ $\mathbb{R}$. This is performed using a path integral over the modes, which generate a quasiassociative algebra. The resulting models have a manifest $U_{q}(s u(2))$ symmetry with a smooth limit $q \rightarrow 1$, and satisfy positivity and twisted bosonic symmetry properties. A systematic way to calculate $n$-point correlators in perturbation theory is given. As examples, the 4 -point correlator for a free scalar field theory and the planar contribution to the tadpole diagram in $\phi^{4}$ theory are computed. The case of gauge fields is also discussed, as well as an operator formulation of scalar field theory in $2_{q}+1$ dimensions. An alternative, essentially equivalent approach using associative techniques only is also presented. The proposed framework is not restricted to 2 dimensions.
\end{abstract}

\footnotetext{
${ }^{1}$ grosse@doppler.thp.univie.ac.at

${ }^{2}$ John.Madore@th.u-psud.fr

${ }^{3}$ Harold.Steinacker@th.u-psud.fr
} 


\section{Contents}

\begin{tabular}{lll}
\hline & Introduction & 1
\end{tabular}

2 Some mathematical background: Drinfel'd twists 4

$\begin{array}{lll}3 & \text { Twisted } U_{q}(g) \text {-covariant } \star \text {-product algebras } & 7\end{array}$

3.1 Integration . . . . . . . . . . . . . . . . . . . 11

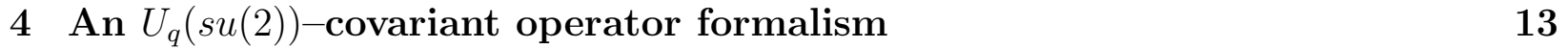

4.1 Reality structure. . . . . . . . . . . . . . . . . . . . . . 16

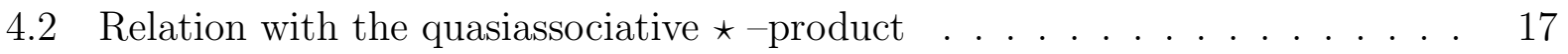

$\begin{array}{llr}5 & \text { Twisted Euclidean QFT } & 18\end{array}$

5.1 Star product approach $\ldots \ldots \ldots$. . . . . . . . . . . . . . . . . 21

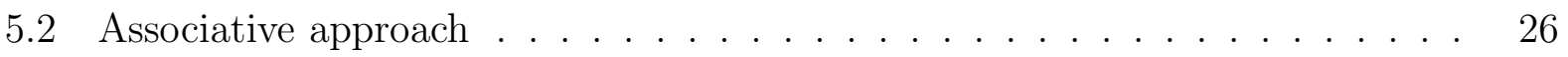

$\begin{array}{lll}6 & \text { Examples } & 30\end{array}$

6.1 The free scalar field . . . . . . . . . . . . . . . . . . . . . . . 30

6.2 Remarks on $N \rightarrow \infty$ and $\phi^{4}$ theory. . . . . . . . . . . . . . . . . . . . . . . 32

6.3 Gauge fields . . . . . . . . . . . . . . . . . . . . . . . . . . . . 33

6.4 QFT in $2_{q}+1$ dimensions, Fock space . . . . . . . . . . . . . 36

\begin{tabular}{|ll}
\hline 7 Appendix A: some proofs & 38 \\
\hline
\end{tabular}

\section{Introduction}

The idea of studying field theory on $q$-deformed spaces has been pursued since their appearance more than 10 years ago. While much work has been done on the level of first quantization (see for example [3, 9, 12, 14, 20, 22, 25] and references therein), the second quantization has proved to be difficult. The main problem is perhaps the apparent incompatibility between the symmetrization postulate of quantum field theory (QFT), and the fact that quantum groups are naturally associated with the braid group rather than the symmetric group. One could of course consider theories with generalized statistics; however if $q$-deformation is considered as a true "deformation" of ordinary space, then it should be 
possible to define models with a smooth limit $q \rightarrow 1$. In particular, the number of degrees of freedom should be independent of $q$. The goal of the present paper is to define a $q$-deformed QFT which is essentially bosonic, and has a smooth limit $q \rightarrow 1$.

In our previous work [12], we studied in detail field theory on the $q$-deformed fuzzy spheres $\mathcal{S}_{q, N}^{2}$ at the first-quantized level. The spheres $\mathcal{S}_{q, N}^{2}$ are precisely the "discrete series" of Podles spheres [24] if $q \in \mathbb{R}$. This space is particularly well suited to attack the problem of second quantization, because there is only a finite number of modes. Therefore all considerations can be done on a purely algebraic level, and are essentially rigorous. The methods we shall develop here are however not restricted to that case, but should generalize immediately to other $q$-deformed spaces, at least on a formal level. There will be complications, of course, if the number of modes is infinite.

To understand the problem, consider scalar fields, which are elements $\psi \in \mathcal{S}_{q, N}^{2}$. A typical action can have the form

$$
S[\psi]=-\int_{\mathcal{S}_{q, N}^{2}} \frac{1}{2} \psi^{*} \Delta \psi+\lambda \psi^{4},
$$

where $\Delta$ is the Laplacian [12]. Such actions are invariant under the quantum group $U_{q}(s u(2))$ of rotations, and they are real, $S[\psi]^{*}=S[\psi]$. They define a first-quantized euclidean scalar field theory on the $q$-deformed fuzzy sphere.

We want to study the second quantization of these models. On the undeformed fuzzy sphere, this is fairly straightforward [13, 19]: The fields can be expanded in terms of irreducible representations of $S O(3)$,

$$
\psi(x)=\sum_{K, n} \psi_{K, n}(x) a^{K, n}
$$

with coefficients $a^{K, n} \in \mathbb{C}$. The above actions then become polynomials in the variables $a^{K, n}$ which are invariant under $S O(3)$, and the "path integral" is naturally defined as the product of the ordinary integrals over the coefficients $a^{K, n}$. This defines a quantum field theory which has a $S O(3)$ rotation symmetry, because the path integral is invariant.

In the $q$-deformed case, this is not so easy. The reason is that the coefficients $a^{K, n}$ in (1.2) must be considered as representations of $U_{q}(s u(2))$ in order to have such a symmetry at the quantum level. This implies that they cannot be ordinary complex numbers, because a commutative algebra is not consistent with the action of $U_{q}(s u(2))$, whose coproduct is not cocommutative. Therefore an ordinary integral over commutative modes $a^{K, n}$ would violate $U_{q}(s u(2))$ invariance at the quantum level. On the other hand, no associative algebra with generators $a^{K, n}$ is known (except for some simple representations) which is both covariant under $U_{q}(s u(2))$ and has the same Poincaré series as classically, i.e. the dimension of the 
space of polynomials at a given degree is the same as in the undeformed case. The latter is an essential physical requirement at least for low energies, in order to have the correct number of degrees of freedom, and is usually encoded in a symmetrization postulate. It means that the "amount of information" contained in the $n$-point functions should be the same as for $q=1$. These issues will be discussed on a more formal level in Section 5. While some proposals have been given in the literature [23, 4] how to define QFT on spaces with quantum group symmetry, none of them seems to satisfies all these requirements.

One possible way out has been suggested in [11], where it was shown that a symmetrization can be achieved using a Drinfel'd twist, at least in any given $n$-particle sector. Roughly speaking, the Drinfel'd twist relates the tensor product of representations of quantum groups to the tensor product of undeformed ones, and hence essentially allows to use the usual completely symmetric Hilbert space. The problem remained, however, how to treat sectors with different particle number simultaneously, which is essential for a QFT, and how to handle the Drinfel'd twists which are very difficult to calculate.

We present here a formalism which solves these problems, by defining a star product of the modes $a^{K, n}$ which is covariant under the quantum group, and in the limit $q \rightarrow 1$ reduces to the commutative algebra of functions in the $a^{K, n}$. This algebra is quasiassociative, but satisfies all the requirements discussed above. In particular, the number of independent polynomials in the $a^{K, n}$ is the same as usual. One can then define an invariant path integral, which yields a consistent and physically reasonable definition of a second-quantized field theory with a quantum group symmetry. In particular, the "correlation functions" will satisfy invariance, hermiticity, positivity and symmetry properties. An essentially equivalent formulation in terms of a slightly extended associative algebra will be presented as well, based on constructions by Fiore [10]. It turns out to be related to the general considerations in [18]. The appearance of quasiassociative algebras is also consistent with results in the context of $D$-branes on WZW models [1].

Our considerations are not restricted to 2 dimensions, and should be applicable to other spaces with quantum group symmetry as well. The necessary mathematical tools will be developed in Sections 2, 3, and \&. After discussing the definition and basic properties of QFT on $\mathcal{S}_{q, N}^{2}$ in Section 5, we derive formulas to calculate $n$-point functions in perturbation theory, and find an analog of Wick's theorem. All diagrams on $\mathcal{S}_{q, N}^{2}$ are of course finite, and vacuum diagrams turn out to cancel a usual. The resulting models can also be interpreted as field theories on the undeformed fuzzy sphere, with slightly "nonlocal" interactions.

As applications of the general method, we consider first the case of a free scalar field theory, and calculate the 4 -point functions. The tadpole diagram for a $\phi^{4}$ model is studied as well, and turns out to be linearly divergent as $N \rightarrow \infty$. We then discuss two possible quantizations of gauge models, and finally consider scalar field theory on $\mathcal{S}_{q, N}^{2}$ with an extra 
time.

We should stress that our approach is quite conservative, as it aims to find a "deformation" of standard quantum field theory in a rather strict sense, with ordinary statistics. Of course on can imagine other, less conventional approaches, such as the one in [23]. Moreover, we only consider the case $q \in \mathbb{R}$ in this paper. It should be possible to modify our methods so that the case of $q$ being a root of unity can also be covered. Then QFT on more realistic spaces such as 4-dimensional quantum Anti-de Sitter space [27] could be considered as well. There, the number of modes as well as the dimensions of the relevant representations are finite at roots of unity, as in the present paper.

\section{Some mathematical background: Drinfel'd twists}

We first review some mathematical results which are the basis of the later considerations. In order to avoid confusions, the language will be quite formal initially. To a given a finitedimensional simple Lie algebra $g$ (for our purpose just $s u(2)$ ), one can associate 2 Hopf algebras: the usual $(U(g)[[h]], m, \varepsilon, \Delta, S)$, and the $q$-deformed $\left(U_{q}(g), m_{q}, \varepsilon_{q}, \Delta_{q}, S_{q}\right)$. Here $U(g)$ is the universal enveloping algebra, $U_{q}(g)$ is the $q$-deformed universal enveloping algebra, and $U(g)[[h]]$ are the formal power series in $h$ with coefficients in $U(g)$. The symbol

$$
q=e^{h}
$$

is considered formal for now. Then a well-known theorem by Drinfel'd (Proposition 3.16 in Ref. [7]) states that there exists an algebra isomorphism

$$
\varphi: U_{q}(g) \rightarrow U(g)[[h]]
$$

and a 'twist', i.e. an element

$$
\mathcal{F}=\mathcal{F}_{1} \otimes \mathcal{F}_{2} \in U(g)[[h]] \otimes U(g)[[h]]
$$

(in a Sweedler notation, where a sum is implicitly understood) satisfying

$$
\begin{aligned}
& (\varepsilon \otimes \mathrm{id}) \mathcal{F}=\mathbf{1}=(\mathrm{id} \otimes \varepsilon) \mathcal{F}, \\
& \mathcal{F}=\mathbf{1} \otimes \mathbf{1}+o(h),
\end{aligned}
$$

which relates these two Hopf algebra $U_{q}(g)$ and $U(g)[[h]]$ as follows: if $\mathcal{F}^{-1}=\mathcal{F}_{1}^{-1} \otimes \mathcal{F}_{2}^{-1}$ is the inverse ${ }^{4}$ of $\mathcal{F}$, then

$$
\varphi\left(m_{q}\right)=m \circ(\varphi \otimes \varphi)
$$

\footnotetext{
${ }^{4}$ it exists as a formal power series because of 2.3
} 


$$
\begin{aligned}
\varepsilon_{q} & =\varepsilon \circ \varphi \\
\varphi\left(S_{q}(u)\right) & =\gamma^{-1} S(\varphi(u)) \gamma \\
\varphi\left(S_{q}^{-1}(u)\right) & =\gamma^{\prime} S(\varphi(u)) \gamma^{\prime-1} \\
(\varphi \otimes \varphi) \Delta_{q}(u) & =\mathcal{F} \Delta(\varphi(u)) \mathcal{F}^{-1} \\
(\varphi \otimes \varphi) \mathcal{R} & =\mathcal{F}_{21} q^{\frac{t}{2}} \mathcal{F}^{-1} .
\end{aligned}
$$

for any $u \in U_{q}(g)$. Here $t:=\Delta(C)-\mathbf{1} \otimes C-C \otimes \mathbf{1}$ is the canonical invariant element in $U(g) \otimes U(g), C$ is the quadratic Casimir, and

$$
\begin{array}{rlrl}
\gamma & =S\left(\mathcal{F}_{1}^{-1}\right) \mathcal{F}_{2}^{-1}, & \gamma^{\prime}=\mathcal{F}_{2} S \mathcal{F}_{1}, \\
\gamma^{-1}=\mathcal{F}_{1} S \mathcal{F}_{2}=S \gamma^{\prime}, & \gamma^{\prime-1}=S\left(\mathcal{F}_{2}^{-1}\right) \mathcal{F}_{1}^{-1}=S \gamma
\end{array}
$$

Moreover, $\gamma^{-1} \gamma^{\prime}$ is central in $U(g)[[h]]$. The undeformed maps $m, \varepsilon, \Delta, S$ have been linearly extended from $U(g)$ to $U(g)[[h]]$; notice that $S^{2}=1$. $\mathcal{F}_{21}$ is obtained from $\mathcal{F}$ by flipping the tensor product. This kind of notation will be used throughout from now on. Coassociativity of $\Delta_{q}$ follows from the fact that the (nontrivial) coassociator

$$
\phi:=\left[(\Delta \otimes \mathrm{id}) \mathcal{F}^{-1}\right]\left(\mathcal{F}^{-1} \otimes \mathbf{1}\right)(\mathbf{1} \otimes \mathcal{F})[(\mathrm{id} \otimes \Delta) \mathcal{F}]
$$

is $U(g)$-invariant, i.e.

$$
\left[\phi, \Delta^{(2)}(u)\right]=0
$$

for $u \in U(g)$. Here $\Delta^{(2)}$ denotes the usual 2-fold coproduct.

In the present paper, we only consider finite-dimensional representations, i.e. operator algebras rather than the abstract ones. Then the formal parameter $q=e^{h}$ can be replaced by a real number close to 1 , and all statements in this section still hold since the power series will converge. One could then identify the algebras $U(s u(2))$ with $U_{q}(s u(2)$ ) (but not as coalgebras!) via the the isomorphism $\varphi$. We will usually keep $\varphi$ explicit, however, in order to avoid confusions.

It turns out that the twist $\mathcal{F}$ is not determined uniquely, but there is some residual "gauge freedom" [7],

$$
\mathcal{F} \rightarrow \mathcal{F} T
$$

with an arbitrary symmetric $T \in U(g)[[h]]^{\otimes 2}$ which commutes with $\Delta(U(g))$ and satisfies (2.2), (2.3). The symmetry of $T$ guarantees that $\mathcal{R}$ is unchanged, so that $\mathcal{F}$ remains a twist from $(U(g)[[h]], m, \varepsilon, \Delta, S)$ to $\left(U_{q}(q), m_{q}, \varepsilon_{q}, \Delta_{q}, S_{q}\right)$. We will take advantage of this below.

\footnotetext{
${ }^{5}$ we will suppress the multiplication maps from now on
} 
While for the twist $\mathcal{F}$, little is known apart apart from its existence, one can show 10 using results of Kohno [17] and Drinfel'd [7] that the twists can be chosen such that the following formula holds:

$$
\phi=\lim _{x_{0}, y_{0} \rightarrow 0^{+}}\left\{x_{0}^{-\frac{h}{2 \pi i} t_{12}} \vec{P} \exp \left[-\frac{h}{2 \pi i} \int_{x_{0}}^{1-y_{0}} d x\left(\frac{t_{12}}{x}+\frac{t_{23}}{x-1}\right)\right] y_{0}^{\frac{h}{2 \pi i} t_{23}}\right\}=\mathbf{1}+o\left(h^{2}\right) .
$$

Here $\vec{P}$ denotes the path-ordered exponential. Such twists were called "minimal" by Fiore [10], who showed that they satisfy the following remarkable relations:

$$
\begin{aligned}
\mathbf{1} & =\mathcal{F} \Delta \mathcal{F}_{1}\left(1 \otimes\left(S \mathcal{F}_{2}\right) \gamma\right), \\
& =\left(1 \otimes S \mathcal{F}_{2} \gamma^{\prime-1}\right) \mathcal{F}\left(\Delta \mathcal{F}_{1}\right) \\
& =\mathcal{F} \Delta \mathcal{F}_{2}\left(\left(S \mathcal{F}_{1}\right) \gamma^{\prime-1} \otimes 1\right) \\
& =\left(\gamma^{-1} S \mathcal{F}_{1}^{-1} \otimes 1\right) \Delta \mathcal{F}_{2}^{-1} \mathcal{F}^{-1} \\
& =\Delta \mathcal{F}_{2}^{-1} \mathcal{F}^{-1}\left(\gamma^{\prime} S \mathcal{F}_{1}^{-1} \otimes 1\right) \\
& =\left(1 \otimes \gamma^{\prime} S \mathcal{F}_{2}^{-1}\right) \Delta \mathcal{F}_{1}^{-1} \mathcal{F}^{-1}
\end{aligned}
$$

All coproducts here are undeformed. Furthermore, we add the following observation: let $\left(V_{i}, \triangleright\right)$ be representations of $U(g)$ and $I^{(3)} \in V_{1} \otimes V_{2} \otimes V_{3}$ be an invariant tensor, so that $u \triangleright I^{(3)} \equiv \Delta^{(2)}(u) \triangleright I^{(3)}=\varepsilon(u) I^{(3)}$ for $u \in U(g)$. Then the (component-wise) action of $\phi$ on $I^{(3)}$ is trivial:

$$
\phi \triangleright I^{(3)}=I^{(3)} .
$$

This follows from (2.13): observe that $t_{12}$ commutes with $t_{23}$ in the exponent, because e.g. $(\Delta(C) \otimes \mathbf{1})$ can be replaced by $\mathbf{1} \otimes \mathbf{1} \otimes C$ if acting on invariant tensors. Therefore the pathordering becomes trivial, and (2.20) follows.

Star structure. Consider on $U(s u(2))[[h]]$ the (antilinear) star structure

$$
H^{*}=H, \quad X^{ \pm *}=X^{\mp}
$$

with $h^{*}=h$, since $q$ is real. It follows e.g. from its explicit form [5] that the algebra map $\varphi$ is compatible with this star,

$$
\varphi(u)^{*}=\varphi\left(u^{*}\right) .
$$

It was shown in [16] that using a suitable gauge transformation (2.12), it is possible to choose $\mathcal{F}$ such that it is unitary,

$$
(* \otimes *) \mathcal{F}=\mathcal{F}^{-1}
$$

Moreover, it was stated in [10] without proof that the following stronger statement holds: 
Proposition 2.1 Using a suitable gauge transformation (2.19), it is possible to choose a twist $\mathcal{F}$ which for $q \in \mathbb{R}$ is both unitary and minimal, so that (2.29) and (2.14) to (2.19) hold.

Since this is essential for us, we provide a proof in Appendix A.

\section{Twisted $U_{q}(g)$-covariant $\star$-product algebras}

Let $(\mathcal{A}, \cdot, \triangleright)$ be an associative $U(g)$-module algebra, which means that there exists an action

$$
\begin{aligned}
U(g) \times \mathcal{A} & \rightarrow \mathcal{A}, \\
(u, a) & \mapsto u \triangleright a
\end{aligned}
$$

which satisfies $u \triangleright(a b)=\left(u_{(1)} \triangleright a\right)\left(u_{(2)} \triangleright b\right)$ for $a, b \in \mathcal{A}$. Here $\Delta(u)=u_{(1)} \otimes u_{(2)}$ denotes the undeformed coproduct. Using the map $\varphi$ (2.11), we can then define an action of $U_{q}(g)$ on $\mathcal{A}$ by

$$
u \triangleright_{q} a:=\varphi(u) \triangleright a,
$$

or $u \triangleright_{q} a_{i}=a_{j} \pi_{i}^{j}(\varphi(u))$ in matrix notation. This does not define a $U_{q}(g)$-module algebra, because the multiplication is not compatible with the coproduct of $U_{q}(g)$. However, one can define a new multiplication on $\mathcal{A}$ as follows:

$$
a \star b:=\left(\mathcal{F}_{1}^{-1} \triangleright a\right) \cdot\left(\mathcal{F}_{2}^{-1} \triangleright b\right)=\cdot\left(\mathcal{F}^{-1} \triangleright(a \otimes b)\right)
$$

for any $a, b \in \mathcal{A}$. It is well-known [21] that $\left(\mathcal{A}, \star, \triangleright_{q}\right)$ is now a $U_{q}(g)$-module algebra:

$$
\begin{aligned}
u \triangleright_{q}(a \star b) & =\varphi(u) \triangleright\left(\left(\mathcal{F}_{1}^{-1} \triangleright a\right) \cdot\left(\mathcal{F}_{2}^{-1} \triangleright b\right)\right) \\
& =\cdot\left(\left(\Delta(\varphi(u)) \mathcal{F}^{-1}\right) \triangleright a \otimes b\right) \\
& =\cdot\left(\left(\mathcal{F}^{-1}(\varphi \otimes \varphi) \Delta_{q}(u)\right) \triangleright a \otimes b\right) \\
& =\star\left(\Delta_{q}(u) \triangleright_{q} a \otimes b\right)
\end{aligned}
$$

for $u \in U_{q}(g)$. In general, this product $\star$ is not associative, but it is quasiassociative, which means that

$$
(a \star b) \star c=\left(\tilde{\phi}_{1} \triangleright a\right) \star\left(\left(\tilde{\phi}_{2} \triangleright b\right) \star\left(\tilde{\phi}_{3} \triangleright c\right)\right) .
$$

where

$$
\tilde{\phi}:=(\mathbf{1} \otimes \mathcal{F})[(\mathrm{id} \otimes \Delta) \mathcal{F}]\left[(\Delta \otimes \mathrm{id}) \mathcal{F}^{-1}\right]\left(\mathcal{F}^{-1} \otimes \mathbf{1}\right)=\mathrm{U}_{\mathcal{F}} \phi \mathrm{U}_{\mathcal{F}}^{-1}
$$


with

$$
U_{\mathcal{F}}=(\mathbf{1} \otimes \mathcal{F})[(\mathrm{id} \otimes \Delta) \mathcal{F}] \quad \in \mathrm{U}(\mathrm{g})^{\otimes 3}
$$

which satisfies

$$
\left[\tilde{\phi}, \Delta_{q}^{(2)}(u)\right]=0
$$

for $u \in U_{q}(g)$. All this follows immediately from the definitions. Moreover, the following simple observation will be very useful:

Lemma 3.1 In the above situation,

$$
(a \star b) \star c=a \star(b \star c)
$$

if one of the factors $a, b, c \in \mathcal{A}$ is invariant under $U(g)$. If $(\mathcal{A}, \cdot)$ is commutative, then any element $S \in \mathcal{A}$ which is invariant under the action of $U(g), u \triangleright S=\varepsilon(u) S$, is central in $\left(\mathcal{A}, \star, \triangleright_{q}\right)$

Note that invariance of an element $a \in \mathcal{A}$ under $U(g)$ is the same as invariance under $U_{q}(g)$. Proof This follows immediately from (2.2) together with the definition of $\tilde{\phi}$. To see the last statement, assume that $S$ is invariant. Then

$$
\begin{aligned}
S \star a & =\left(\mathcal{F}_{1}^{-1} \triangleright S\right) \cdot\left(\mathcal{F}_{2}^{-1} \triangleright a\right) \\
& =\cdot\left(\left((\varepsilon \otimes \mathbf{1}) \mathcal{F}^{-1}\right) \triangleright(S \otimes a)\right) \\
& =S \cdot a=a \cdot S \\
& =a \star S
\end{aligned}
$$

for any $a \in \mathcal{A}$.

For actual computations, it is convenient to use a tensor notation as follows: assume that the elements $\left\{a_{i}\right\}$ of $\mathcal{A}$ form a representation of $U(g)$. Denoting $\tilde{\phi}_{i j k}^{r s t}=\pi_{i}^{r}\left(\tilde{\phi}_{1}\right) \pi_{j}^{s}\left(\tilde{\phi}_{2}\right) \pi_{k}^{t}\left(\tilde{\phi}_{3}\right)$, equation (3.3) can be written as

$$
\begin{aligned}
& \left(a_{i} \star a_{j}\right) \star a_{k}=a_{r} \star\left(a_{s} \star a_{t}\right) \tilde{\phi}_{i j k}^{r s t}, \text { or } \\
& \left(a_{1} \star a_{2}\right) \star a_{3}=a_{1} \star\left(a_{2} \star a_{3}\right) \tilde{\phi}_{123} .
\end{aligned}
$$

The last notation will always imply a matrix multiplication as above.

Conversely, given a $U_{q}(g)$-module algebra $\left(\mathcal{A}, \star, \triangleright_{q}\right)$, one can twist it into a $U(g)$-module algebra $(\mathcal{A}, \cdot, \triangleright)$ by

$$
a \cdot b:=\left(\varphi^{-1}\left(\mathcal{F}^{(1)}\right) \triangleright_{q} a\right) \star\left(\varphi^{-1}\left(\mathcal{F}^{(2)}\right) \triangleright_{q} b\right)
$$


where of course $u \triangleright a=\varphi^{-1}(u) \triangleright_{q} a$. Now if $\left(\mathcal{A}, \star, \triangleright_{q}\right)$ was associative, then $(\mathcal{A}, \cdot, \triangleright)$ is quasiassociative,

$$
a \cdot(b \cdot c)=\phi \triangleright_{q}^{(3)}((a \cdot b) \cdot c):=\left(\left(\phi_{1} \triangleright_{q} a\right) \cdot\left(\phi_{2} \triangleright_{q} b\right)\right) \cdot\left(\phi_{3} \triangleright_{q} c\right) .
$$

Such a twist was used in 12 to obtain the associative algebra of functions on the $q$-deformed fuzzy sphere from the quasi-associative algebra of functions on $D 2$-branes in the $S U(2)$ WZW model found in [1].

Commutation relations and $\mathcal{R}$-matrices. These twisted algebras have a more intrinsic characterization, which is much more practical. Consider a commutative $U(g)$-module algebra $(\mathcal{A}, \cdot, \triangleright)$, and the associated twisted $U_{q}(g)$-module algebra $\left(\mathcal{A}, \star, \triangleright_{q}\right)$ as defined above. Observe that the definition (3.2) is equivalent to

$$
\begin{aligned}
a \star b & =\left(\mathcal{F}_{1}^{-1} \triangleright a\right) \cdot\left(\mathcal{F}_{2}^{-1} \triangleright b\right)=\left(\mathcal{F}_{2}^{-1} \triangleright b\right) \cdot\left(\mathcal{F}_{1}^{-1} \triangleright a\right) \\
& =\cdot\left(\left(\mathcal{F}^{-1} \mathcal{F} \mathcal{F}_{21}^{-1}\right) \triangleright(b \otimes a)\right) \\
& =\left(\tilde{\mathcal{R}}_{2} \triangleright_{q} b\right) \star\left(\tilde{\mathcal{R}}_{1} \triangleright_{q} a\right)
\end{aligned}
$$

where we define

$$
\tilde{\mathcal{R}}:=\left(\varphi^{-1} \otimes \varphi^{-1}\right) \mathcal{F}_{21} \mathcal{F}^{-1}=\tilde{\mathcal{R}}_{21}^{-1}
$$

In a given representation, this can be written as

$$
a_{i} \star a_{j}=a_{k} \star a_{l} \tilde{\mathcal{R}}_{i j}^{l k}, \quad \text { or } \quad a_{1} \star a_{2}=a_{2} \star a_{1} \tilde{\mathcal{R}}_{12}
$$

where

$$
\tilde{\mathcal{R}}_{k l}^{j i}=\left(\pi_{k}^{j} \otimes \pi_{l}^{i}\right)(\tilde{\mathcal{R}}) .
$$

Now there is no more reference to the "original" $U(g)$-covariant algebra structure. $\tilde{\mathcal{R}}$ does not satisfy the quantum Yang-Baxter equation in general, which reflects the non-associativity of the $\star$ product. However it does satisfy

$$
\begin{aligned}
\tilde{\mathcal{R}} \tilde{\mathcal{R}}_{21} & =\mathbf{1} \\
\tilde{\mathcal{R}}_{(12), 3}:=\left(\Delta_{q} \otimes 1\right) \tilde{\mathcal{R}} & =\tilde{\phi}_{312} \tilde{\mathcal{R}}_{13} \tilde{\phi}_{132}^{-1} \tilde{\mathcal{R}}_{23} \tilde{\phi}_{123} \\
\tilde{\mathcal{R}}_{1,(23)}:=\left(1 \otimes \Delta_{q}\right) \tilde{\mathcal{R}} & =\tilde{\phi}_{231}^{-1} \tilde{\mathcal{R}}_{13} \tilde{\phi}_{213} \tilde{\mathcal{R}}_{12} \tilde{\phi}_{123}^{-1},
\end{aligned}
$$

as can be verified easily. This means that we are working with the quasitriangular quasi-Hopf algebra [7] $\left(U_{q}(g), \Delta_{q}, \tilde{\phi}, \tilde{\mathcal{R}}\right)$, which is obtained from the ordinary Hopf algebra $(U(g), \Delta, \mathbf{1}, \mathbf{1})$ 
by the Drinfel'd twist $\mathcal{F}$. In practice, it is much easier to work with $\tilde{\mathcal{R}}$ than with $\mathcal{F}$. For $q \in \mathbb{R}$, one can in fact write

$$
\tilde{\mathcal{R}}=\mathcal{R}{\sqrt{\mathcal{R}_{21} \mathcal{R}_{12}}}^{-1}
$$

where $\mathcal{R}$ is the usual universal $R$-matrix (2.9) of $U_{q}(g)$, which does satisfy the quantum Yang-Baxter equation. The product $\left(\mathcal{R}_{21} \mathcal{R}_{12}\right)$ could moreover be expressed in terms of the Drinfel'd-Casimir

$$
v=\left(S_{q} \mathcal{R}_{2}\right) \mathcal{R}_{1} q^{-H},
$$

which is central in $U_{q}(g)$ and satisfies $\Delta(v)=\left(\mathcal{R}_{21} \mathcal{R}_{12}\right)^{-1} v \otimes v$. The square root is welldefined on all the representations which we consider, since $q$ is real.

Twisted Heisenberg algebras. Consider the $U(g)$-module algebra $\left(\mathcal{A}_{H}, \cdot, \triangleright\right)$ with generators $a_{i}$ and $a_{j}^{\dagger}$ in some given irreducible representation and commutation relations

$$
\begin{aligned}
{\left[a_{i}^{\dagger}, a_{j}^{\dagger}\right] } & =0=\left[a_{i}, a_{j}\right] \\
{\left[a_{i}^{\dagger}, a_{j}\right] } & =\left(g_{c}\right)_{i j}
\end{aligned}
$$

where $\left(g_{c}\right)_{i j}$ is the (unique) invariant tensor in the given representation of $U(g)$. We can twist $\left(\mathcal{A}_{H}, \cdot, \triangleright\right)$ as above, and obtain the $U_{q}(g)$-module algebra $\left(\mathcal{A}_{H}, \star, \triangleright_{q}\right)$. The new commutation relations among the generators can be evaluated easily:

$$
\begin{aligned}
a_{1} \star a_{2} & =a_{2} \star a_{1} \tilde{\mathcal{R}}_{12}, \\
a_{1}^{\dagger} \star a_{2}^{\dagger} & =a_{2}^{\dagger} \star a_{1}^{\dagger} \tilde{\mathcal{R}}_{12}, \\
a_{1}^{\dagger} \star a_{2} & =g_{12}+a_{2} \star a_{1}^{\dagger} \tilde{\mathcal{R}}_{12} .
\end{aligned}
$$

Here

$$
g_{n m}=\left(g_{c}\right)_{r s} \pi_{n}^{r}\left(\mathcal{F}_{1}^{-1}\right) \pi_{m}^{s}\left(\mathcal{F}_{2}^{-1}\right)
$$

is the unique rank 2 tensor which is invariant under the action $\triangleright_{q}$ of $U_{q}(g)$. A similar relation holds for the invariant tensor with upper indices:

$$
g^{n m}=\pi_{r}^{n}\left(\mathcal{F}_{1}\right) \pi_{s}^{m}\left(\mathcal{F}_{2}\right) g_{c}^{r s},
$$

which satisfies $g^{n m} g_{m l}=\delta_{l}^{n}$. In particular, it follows that

$$
a \star a:=a_{1} \star a_{2} g^{12}=a_{1} \cdot a_{2}\left(g_{c}\right)^{12},
$$

therefore the invariant bilinears remain undeformed. This is independent of the algebra of the generators $a_{i}$. 
It is sometimes convenient to use the $q$-deformed antisymmetrizer [11]

$$
P_{12}^{-}=\mathcal{F}_{12}\left(1-\delta_{12}^{21}\right) \mathcal{F}_{12}^{-1}=(\mathbf{1}-P \tilde{\mathcal{R}})_{12}
$$

acting on the tensor product of 2 identical representations, where $P$ is the flip operator. Then the commutation relations (3.10) can be written as

$$
a_{1} \star a_{2} P_{12}^{-}=0
$$

For products of 3 generators, the following relations hold:

\section{Lemma 3.2}

$$
\begin{aligned}
a_{1} \star\left(a_{2} \star a_{3}\right) & =\left(a_{2} \star a_{3}\right) \star a_{1} \tilde{\mathcal{R}}_{1,(23)}, \\
a_{1}^{\dagger} \star\left(a_{2} \star a_{3} g^{23}\right) & =2 a_{1}+\left(a_{2} \star a_{3} g^{23}\right) \star a_{1}^{\dagger}, \\
a_{1}^{\dagger} \star\left(a_{2} \star a_{3} P_{23}^{-}\right) & =\left(a_{2} \star a_{3} P_{23}^{-}\right) \star a_{1}^{\dagger} \tilde{\mathcal{R}}_{1,(23)} .
\end{aligned}
$$

The proof is in the Appendix.

\subsection{Integration}

From now on we specialize to $g=s u(2)$, even though much of the following holds more generally. Let $\left\{a_{i}\right\}$ be a basis of the spin $K$ representation of $U(s u(2))$ with integer $K$, and consider the (free) commutative algebra $\mathcal{A}$ generated by these variables. Let $g_{c}^{i j}$ be the (real, symmetric) invariant tensor, so that $a \cdot a:=a_{i} a_{j} g_{c}^{i j}$ is invariant under $U(s u(2))$, and $g_{c}^{i j} g_{c}^{j k}=\delta^{i k}$. We now impose on $\mathcal{A}$ the star structure

$$
a_{i}^{*}=g_{c}^{i j} a_{j}
$$

so that $\mathcal{A}$ can be interpreted as the algebra of complex-valued functions on $\mathbb{R}^{2 K+1}$; in particular, $a \cdot a$ is real. Then the usual integral on $\mathbb{R}^{2 K+1}$ defines a functional on (the subset of integrable functions in a suitable completion of) $\mathcal{A}$, which satisfies

$$
\begin{aligned}
\int d^{2 K+1} a u \triangleright f & =\varepsilon(u) \int d^{2 K+1} a f \\
\left(\int d^{2 K+1} a f\right)^{*} & =\int d^{2 K+1} a f^{*}
\end{aligned}
$$


for $u \in U(s u(2))$ and integrable $f \in \mathcal{A}$. More general invariant functionals on $\mathcal{A}$ can be defined as

$$
\langle f\rangle:=\int d^{2 K+1} a \rho(a \cdot a) f
$$

for $f \in \mathcal{A}$, where $\rho$ is a suitable real weight function. They are invariant, real and positive:

$$
\begin{aligned}
\langle u \triangleright f\rangle & =\varepsilon(u)\langle f\rangle, \\
\langle f\rangle^{*} & =\left\langle f^{*}\right\rangle \\
\left\langle f^{*} f\right\rangle & \geq 0
\end{aligned}
$$

for any $u \in U(s u(2))$ and $f \in \mathcal{A}$. As usual, one can then define a Hilbert space of square(weight-) integrable functions by

$$
\langle f, g\rangle:=\left\langle f^{*} g\right\rangle=\int d^{2 K+1} a \rho(a \cdot a) f^{*} g .
$$

Now consider the twisted $U_{q}(s u(2))$-module algebra $\left(\mathcal{A}, \star, \triangleright_{q}\right)$ defined in the previous section. We want to find an integral on $\mathcal{A}$ which is invariant under the action $\triangleright_{q}$ of $U_{q}(s u(2))$. Formally, this is very easy: since the space $\mathcal{A}$ is unchanged by the twisting, we can simply use the classical integral again, and verify invariance

$$
\int d^{2 K+1} a u \triangleright_{q} f=\int d^{2 K+1} a \varphi(u) \triangleright f=\varepsilon(\varphi(u)) \int d^{2 K+1} a f=\varepsilon_{q}(u) \int d^{2 K+1} a f .
$$

Notice that the algebra structure of $\mathcal{A}$ does not enter here at all. The compatibility with the reality structure will be discussed in the next section.

Of course we have to restrict to certain classes of integrable functions. However, this is not too hard in the cases of interest. Consider for example the space of Gaussian functions, i.e. functions of the form $P\left(a_{i}\right) e^{-c(a \cdot a)}$ with suitable (polynomial, say) $P\left(a_{i}\right)$. Using (3.21), this is the same as the space of Gaussian functions in the sense of the star product, $P_{\star}\left(a_{i}\right) e^{-c(a \star a)}$. This will imply that all integrals occuring in perturbation theory are well-defined. Furthermore, one can obtain a twisted sphere by imposing the relation $a \star a=a \cdot a=R^{2}$. On this sphere, the integral is well-defined for any polynomial functions. The integral over the twisted $\mathbb{R}^{2 K+1}$ can hence be calculated by first integrating over the sphere and then over the radius. Finally, we point out the following obvious fact:

$$
\langle P(a)\rangle=\left\langle P_{0}(a)\right\rangle
$$

where $P_{0}(a) \in \mathcal{A}$ is the singlet part of the decomposition of the polynomial $P(a)$ under the action $\triangleright_{q}$ of $U_{q}(s u(2))$, or equivalently under the action $\triangleright$ of $U(s u(2))$. 


\section{An $U_{q}(s u(2))$-covariant operator formalism}

In the previous section, we defined quasi-associative algebras of functions on arbitrary representation spaces of $U_{q}(s u(2))$. We will apply this to the coefficients of the fields on $\mathcal{S}_{q, N}^{2}$ later. However, there is an alternative approach within the framework of ordinary operators and representations, which is essentially equivalent for our purpose. We shall follow here closely the constructions in [10]. It seems that both approaches have their own advantages, therefore we want to discuss them both.

We first recall the notion of the semidirect product (cross-product) algebra, which is useful here. Let $(\mathcal{A}, \cdot, \triangleright)$ be an associative $U(s u(2))$-module algebra. Then $U(s u(2)) \ltimes \mathcal{A}$ is the vector space $\mathcal{A} \otimes U(s u(2))$, equipped with the structure of an associative algebra defined by $u a=\left(u_{(1)} \triangleright a\right) u_{(2)}$. Here $u_{(1)} \otimes u_{(2)}$ is the undeformed coproduct of $U(s u(2))$.

In the following, we shall be interested in representations of $\mathcal{A}$ which have a "vacuum" vector $>$ such that all elements can be written in the form $\mathcal{A}\rangle$, i.e. by acting with $\mathcal{A}$ on the vacuum vector. In particular, we will denote with $V_{\mathcal{A}}$ the free left $\mathcal{A}$-module $\left.\mathcal{A}\right\rangle$ which as a vector space is equal to $\mathcal{A}$. This will be called the the "left vacuum representation" (or left regular representation). Now any such representation of $\mathcal{A}$ can naturally be viewed as a representation of $U(s u(2)) \ltimes \mathcal{A}$, if one declares the vacuum vector $\rangle$ to be a singlet under $U(s u(2))$

$$
u\rangle=\varepsilon(u)\rangle
$$

and $u \triangleright(a\rangle)=(u \triangleright a)\rangle$. One can then verify the relations of $U(s u(2)) \ltimes \mathcal{A}$.

Inspired by [10], we define for any $a \in \mathcal{A}$ the element

$$
\hat{a}:=\left(\mathcal{F}_{1}^{-1} \triangleright a\right) \mathcal{F}_{2}^{-1} \in U(s u(2)) \ltimes \mathcal{A} .
$$

Using the definition of the Drinfel'd twist, it is immediate to verify the following properties:

$$
\begin{aligned}
\hat{a}\rangle & =a\rangle \\
\hat{a} \hat{b}\rangle & =(a \star b)\rangle
\end{aligned}
$$

where $(a \star b)$ is the twisted multiplication on $\mathcal{A}$ defined in (3.2). More generally,

$$
\left.\left.\hat{a}_{1} \hat{a}_{2} \ldots . \hat{a}_{k}\right\rangle=\left(a_{1} \star\left(a_{2} \star\left(\ldots a_{k-1} \star a_{k}\right) \ldots\right)\right)\right\rangle
$$

for any $a_{i} \in \mathcal{A}$. Hence the elements $\hat{a}$ realize the twisted product (3.2) on $\mathcal{A}$, with this particular bracketing. If $c \in \mathcal{A}$ is a singlet, or equivalently $[c, U(s u(2))]=0$ in $U(s u(2)) \ltimes \mathcal{A}$, then

$$
\hat{c}=c .
$$

\footnotetext{
${ }^{6}$ provided the kernel of the representation is invariant under $U(s u(2))$, which we shall assume.
} 
If in addition the algebra $\mathcal{A}$ is commutative, then $\hat{c}$ is central in $U(s u(2)) \ltimes \mathcal{A}$. Moreover, the new variables $\hat{a}_{i}$ are automatically covariant under the quantum group $U_{q}(s u(2))$, with the $q$-deformed coproduct: denoting

$$
\hat{u}:=\varphi(u) \in U(s u(2))
$$

for $u \in U_{q}(s u(2))$, one easily verifies

$$
\hat{u} \hat{a}=\widehat{u_{1} \triangleright_{q} a} \widehat{u_{2}},
$$

where $u_{1} \otimes u_{2}$ denotes the $q$-deformed coproduct. In particular,

$$
\begin{aligned}
\hat{u} \hat{a}\rangle & \left.\left.=u \triangleright_{q} a\right\rangle=\widehat{u \triangleright_{q} a}\right\rangle \\
\hat{u} \hat{a} \hat{b}\rangle & \left.=\left(\widehat{u_{1} \triangleright_{q} a}\right)\left(\widehat{u_{2} \triangleright_{q} b}\right)\right\rangle .
\end{aligned}
$$

Therefore $U_{q}(s u(2))$ acts correctly on the $\hat{a}$-variables in the left vacuum representation. More explicitly, assume that $\mathcal{A}$ is generated (as an algebra) by generators $a_{i}$ transforming in the spin $K$ representation $\pi$ of $U(s u(2))$, so that $u a_{i}=a_{j} \pi_{i}^{j}\left(u_{(1)}\right) u_{(2)}$. Then (4.5) becomes

$$
\hat{u} \hat{a}_{i}=\hat{a}_{j} \pi_{i}^{j}\left(\widehat{u}_{1}\right) \widehat{u}_{2} .
$$

In general, the generators $\hat{a}_{i}$ will not satisfy closed commutation relations, even if the $a_{i}$ do. However if $\left[a_{i}, a_{j}\right]=0$, then one can verify that (cp. [10])

$$
\hat{a}_{i} \hat{a}_{j}=\hat{a}_{k} \hat{a}_{l} \Re_{i j}^{l k}
$$

where

$$
\mathfrak{R}_{k l}^{i j}=\left(\pi_{k}^{i} \otimes \pi_{l}^{j} \otimes i d\right)\left(\tilde{\phi}_{213} \tilde{R}_{12} \tilde{\phi}_{123}^{-1}\right) \quad \in U(s u(2)) .
$$

Again, this involves only the coassociator and the universal $\mathcal{R}$-matrix. Such relations for field operators were already proposed in [18] on general grounds; here, they follow from the definition (4.1). In the case of several variables, one finds

$$
\hat{a}_{i} \hat{b}_{j}=\hat{b}_{k} \hat{a}_{l} \mathfrak{R}_{i j}^{l k}
$$

Indeed, no closed quadratic commutation relations for deformed spaces of function with generators $a_{i}$ in arbitrary representations of $U_{q}(s u(2))$ are known, which has has been a major obstacle for defining QFT's on $q$-deformed spaces. In the present approach, the generators $\hat{a}_{i}$ satisfy quadratic commutation relations which close only in the bigger algebra $U(s u(2)) \ltimes \mathcal{A}$. In general, they are not easy to work with. However some simplifications occur if we use minimal twists $\mathcal{F}$ as defined in Section 2, as was observed by Fiore [10]: 
Proposition 4.1 For minimal twists $\mathcal{F}$ as in (2.13), the following relation holds:

$$
g^{i j} \hat{a}_{i} \hat{a}_{j}=g_{c}^{i j} a_{i} a_{j} .
$$

Here

$$
g^{i j}=\pi_{r}^{i}\left(\mathcal{F}_{1}\right) \pi_{s}^{j}\left(\mathcal{F}_{2}\right) g_{c}^{r s}=g_{c}^{i l} \pi_{l}^{j}\left(\gamma^{\prime}\right),
$$

where $\gamma^{\prime}$ is defined in (2.10). In particular if $\mathcal{A}$ is abelian, this implies that $g^{j k} \hat{a}_{j} \hat{a}_{k}$ is central in $U(\operatorname{su}(2)) \ltimes \mathcal{A}$.

We include a short proof in the appendix for convenience. This will be very useful to define a quantized field theory. From now on, we will always assume that the twists are minimal.

Derivatives. Let $\mathcal{A}$ be again the free commutative algebra with generators $a_{i}$ in the spin $K$ representation of $U(s u(2))$, and consider the left vacuum representation $\left.V_{\mathcal{A}}=\mathcal{A}\right\rangle$ of $U(s u(2)) \ltimes \mathcal{A}$. Let $\partial_{i}$ be the (classical) derivatives, which act as usual on the functions in $\mathcal{A}$. They can be considered as operators acting on $V_{\mathcal{A}}$, and as such they satisfy the relations of the classical Heisenberg algebra, $\partial_{i} a_{j}=g_{i j}^{c}+a_{j} \partial_{i}$. Now define

$$
\hat{\partial}_{i}:=\left(\mathcal{F}_{1}^{-1} \triangleright \partial_{i}\right) \mathcal{F}_{2}^{-1},
$$

which is an operator acting on $\left.V_{\mathcal{A}}=\mathcal{A}\right\rangle$; in particular, it satisfies $\left.\hat{\partial}_{i}\right\rangle=0$. Then the following relations hold:

Proposition 4.2 For minimal $\mathcal{F}$ as in (2.13), the operators $\hat{a}_{i}, \hat{\partial}_{j}$ acting on the left vacuum representation satisfy

$$
\begin{aligned}
\hat{\partial}_{i}\left(g^{j k} \hat{a}_{j} \hat{a}_{k}\right) & =2 \hat{a}_{i}+\left(g^{j k} \hat{a}_{j} \hat{a}_{k}\right) \hat{\partial}_{i}, \\
\hat{\partial}_{i} \hat{a}_{j} & =g_{i j}+\hat{a}_{k} \hat{\partial}_{l} \mathfrak{R}_{i j}^{l k} .
\end{aligned}
$$

The proof is given in the appendix; the second relation (4.14) is again very close to a result (Proposition 6) in [10], and it holds in fact in $U(s u(2)) \ltimes \mathcal{A}$. Of course, the brackets in (4.13) were just inserted for better readability, unlike in Lemma 3.2 where they were essential. If we have algebras with several variables in the same representation, then for example

$$
\hat{\partial}_{a_{i}}\left(g^{j k} \hat{b}_{j} \hat{a}_{k}\right)=\hat{b}_{i}+\left(g^{j k} \hat{b}_{j} \hat{a}_{k}\right) \hat{\partial}_{a_{i}}
$$

holds, in self-explanatory notation.

One advantage of this approach compared to the quasi-associative formalism in the previous section is that the concept of a star is clear, induced from Hilbert space theory. This will be explained next. 


\subsection{Reality structure.}

Even though the results of this section are more general, we assume for simplicity that $\mathcal{A}$ is the free commutative algebra generated by the elements $\left\{a_{i}\right\}$ which transform in the spin $K$ representation of $U(s u(2))$ with integer $K$, i.e. the algebra of complex-valued functions on $\mathbb{R}^{2 K+1}$ (or products thereof). Then the classical integral defines an invariant positive functional on $\mathcal{A}$ which satisfies (3.29), and $V_{\mathcal{A}}$ becomes a Hilbert space (3.30) (after factoring out a null space if necessary). Hence we can calculate the operator adjoint of the generators of this algebra. By construction,

$$
a_{i}^{*}=g_{c}^{i j} a_{j}
$$

where $g_{c}^{i j}$ is the invariant tensor, normalized such that $g_{c}^{i j} g_{c}^{j k}=\delta^{i k}$. As discussed above, $V_{\mathcal{A}}$ is a representation of the semidirect product $U(s u(2)) \ltimes \mathcal{A}$, in particular it is a unitary representation of $U(s u(2))$. Hence the star on the generators of $U(s u(2))$ is

$$
H^{*}=H, \quad X^{ \pm *}=X^{\mp} .
$$

Now one can simply calculate the star of the twisted variables $\hat{a}_{i} \in U(s u(2)) \ltimes \mathcal{A}$. The result is as expected:

Proposition 4.3 If $\mathcal{F}$ is a minimal unitary twist as in Proposition 2.1, then the adjoint of the operator $\hat{a}_{i}$ acting on the left vacuum representation $\left.\mathcal{A}\right\rangle$ is

$$
\hat{a}_{i}^{*}=g^{i j} \hat{a}_{j}
$$

This is proved in the appendix, and it was already found in 10. It is straightforward to extend these results to the case of several variables $a_{i}^{(K)}, b_{j}^{(L)}, \ldots$ in different representations, using a common vacuum $\rangle$. The star structure is always of the form (4.3).

If $\mathcal{A}$ is the algebra of functions on $\mathbb{R}^{2 K+1}$, we have seen above that the left vacuum representation $\mathcal{A}\rangle$ is also a representation of the Heisenberg algebra $\mathcal{A}_{H}$ with generators $a_{i}, \partial_{j}$. Again we can calculate the operator adjoints, and the result is

$$
\begin{aligned}
& \partial_{i}^{*}=-g_{c}^{i j} \partial_{j}, \\
& \hat{\partial}_{i}^{*}=-g^{i j} \hat{\partial}_{j} .
\end{aligned}
$$

Of course, all these statements are on a formal level, ignoring operator-technical subtleties. 


\subsection{Relation with the quasiassociative $\star$-product}

Finally, we make a simple but useful observation, which provides the connection of the operator approach in this section with the quasiassociative approach of Section 3. Observe first that an invariant (real, positive (3.29)) functional \langle\rangle on $\mathcal{A}$ extends trivially as a (real, positive) functional on $U(s u(2)) \ltimes \mathcal{A}$, by evaluating the generators of $U(s u(2))$ on the left (or right) of $\mathcal{A}$ with the counit. Now for any tensor $I^{i_{1} \ldots i_{k}}$ of $U_{q}(s u(2))$, denote

$$
I(\hat{a}):=I^{i_{1} \ldots i_{k}} \hat{a}_{i_{1}} \ldots \hat{a}_{i_{k}} \quad \in U(s u(2)) \ltimes \mathcal{A},
$$

and

$$
I_{\star}(a):=I^{i_{1} \ldots i_{k}} a_{i_{1}} \star\left(\ldots \star\left(a_{i_{k-1}} \star a_{i_{k}}\right) \ldots\right) \quad \in \mathcal{A} .
$$

Then the following holds:

Lemma 4.4 1) If $I=I^{i_{1} \ldots i_{k}}$ is an invariant tensor of $U_{q}(s u(2))$, then $I(\hat{a})$ as defined above commutes with $u \in U_{q}(s u(2))$,

$$
[u, I(\hat{a})]=0 \quad \text { in } U(s u(2)) \ltimes \mathcal{A} .
$$

2) Let $s\rangle \in \mathcal{A}\rangle$ be invariant, i.e. $\left.u \cdot s\rangle=\varepsilon_{q}(u) s\right\rangle$, and $I, \ldots, J$ be invariant tensors of $U_{q}(s u(2))$. Then

$$
\left.I(\hat{a}) \ldots J(\hat{a}) s\rangle=I_{\star}(a) \star \ldots \star J_{\star}(a) s\right\rangle .
$$

3) Let $I, J$ be invariant, and $P=P^{i_{1} \ldots i_{k}}$ be an arbitrary tensor of $U_{q}(s u(2))$. Denote with $P_{0}$ the trivial component of $P$ under the action of $U_{q}(s u(2))$. Then for any invariant functional \langle\rangle on $\mathcal{A}$,

$$
\begin{aligned}
\langle I(\hat{a}) \ldots J(\hat{a}) P(\hat{a})\rangle & =\left\langle I(\hat{a}) \ldots J(\hat{a}) P_{0}(\hat{a})\right\rangle \\
& =\left\langle I_{\star}(a) \star \ldots \star J_{\star}(a)\left(P_{0}\right)_{\star}(a)\right\rangle \\
& =\left\langle I_{\star}(a) \star \ldots \star J_{\star}(a) \star P_{\star}(a)\right\rangle
\end{aligned}
$$

Moreover if $\mathcal{A}$ is abelian, then the $I(\hat{a}), J(\hat{a})$ etc. can be considered as central in an expression of this form, for example

$$
\left\langle I(\hat{a}) \ldots J(\hat{a}) P\left(\hat{a}_{i}\right)\right\rangle=\left\langle P\left(\hat{a}_{i}\right) I(\hat{a}) \ldots J(\hat{a})\right\rangle=\left\langle P\left(\hat{a}_{i}\right) J(\hat{a}) \ldots I(\hat{a})\right\rangle
$$

and so on.

The proof follows easily from (4.5), (4.3) and Lemma 3.1. The stars between the invariant polynomials $I_{\star}(a), \ldots, J_{\star}(a)$ are of course trivial, and no brackets are needed. 


\section{Twisted Euclidean QFT}

These tools can now be applied to our problem of quantizing fields on the $q$-deformed fuzzy sphere $\mathcal{S}_{q, N}^{2}$. Most of the discussion is not restricted to this space, but it is on a much more rigorous level there because the number of modes is finite. We will present 2 approaches, the first based on twisted $\star$-products as defined in Section 3, and the second using an operator formalism as in Section 4 . Both have their own merits which seem to justify presenting them both. Their equivalence will follow from Lemma 4.4.

First, we discuss some basic requirements for a quantum field theory on spaces with quantum group symmetry. Consider a scalar field, and expand it in its modes as

$$
\Psi(x)=\sum_{K, n} \psi_{K, n}(x) a^{K, n}
$$

Here the $\psi_{K, n}(x) \in \mathcal{S}_{q, N}^{2}$ are a basis of the spin $K$ representation of $U_{q}(s u(2))$,

$$
u \triangleright_{q} \psi_{K, n}(x)=\psi_{K, m}(x) \pi_{n}^{m}(u)
$$

and the coefficients $a^{K, n}$ transform in the dual (contragredient) representation of $\tilde{U}_{q}(s u(2))$,

$$
u \tilde{\triangleright}_{q} a^{K, n}=\pi_{m}^{n}(\tilde{S} u) a^{K, m} .
$$

It is important to distinguish the Hopf algebras which act on the coefficients $a^{K, n}$ and on the functions $\psi_{K, n}(x)$, respectively. The Hopf algebra $\tilde{U}_{q}(s u(2))$ is obtained from $U_{q}(s u(2))$ by flipping the coproduct and using the opposite antipode $\tilde{S}=S^{-1}$. In particular, the $\mathcal{R}$-matrix and the invariant tensors are also flipped:

$$
\tilde{g}_{n m}^{K}=g_{m n}^{K}
$$

where $\tilde{g}_{n m}^{K}$ is the invariant tensor of $\tilde{U}_{q}(s u(2))$. The reason for this will become clear soon. Moreover, it is sometimes convenient to express the contragredient generators in terms of "ordinary" ones,

$$
a_{K, n}=\tilde{g}_{n m}^{K} a^{K, m}
$$

Then $\tilde{U}_{q}(s u(2))$ acts as

$$
u \tilde{\triangleright}_{q} a_{K, n}=a_{K, m} \pi_{n}^{m}(u) .
$$

We assume that the coefficients $a^{K, n}$ generate some algebra $\mathcal{A}$. This is not necessarily the algebra of field operators, which in fact would not be appropriate in the Euclidean case even for $q=1$. Rather, $\mathcal{A}$ could be the algebra of coordinate functions on configuration space 
(space of modes) for $q=1$, and an analog thereof for $q \neq 1$. The fields $\Psi(x)$ can then be viewed as "algebra-valued distributions" in analogy to usual field theory, by defining

$$
\Psi[f]:=\int_{\mathcal{S}_{q, N}^{2}} \Psi(x) f(x) \quad \in \mathcal{A}
$$

for $f(x) \in \mathcal{S}_{q, N}^{2}$. Then the covariance properties (5.2) and (5.3) could be stated as

$$
u \tilde{\triangleright}_{q} \Psi[f]=\Psi\left[u \triangleright_{q} f\right]
$$

using the fact that $\int\left(u \triangleright_{q} f\right) g=\int f(S(u) \triangleright g)$.

Our goal is to define some kind of correlation functions of the form

$$
\left\langle\Psi\left[f_{1}\right] \Psi\left[f_{2}\right] \ldots \Psi\left[f_{k}\right]\right\rangle \quad \in \mathbb{C}
$$

for any $f_{1}, \ldots, f_{k} \in \mathcal{S}_{q, N}^{2}$, in analogy to the undeformed case. After "Fourier transformation" (5.1), this amounts to defining objects

$$
G^{K_{1}, n_{1} ; K_{2}, n_{2} ; \ldots ; K_{k}, n_{k}}:=\left\langle a^{K_{1}, n_{1}} a^{K_{2}, n_{2}} \ldots a^{K_{k}, n_{k}}\right\rangle=:\langle P(a)\rangle
$$

where $P(a)$ will denote some polynomial in the $a^{K, n}$ from now on, perhaps by some kind of a "path integral" $\langle P(a)\rangle=\frac{1}{\mathcal{N}} \int \mathcal{D} a e^{-S[\Psi]} P(a)$. We require that they should satisfy at least the following properties, to be made more precise later:

(1) Covariance:

$$
\left\langle u \tilde{\triangleright}_{q} P(a)\right\rangle=\varepsilon_{q}(u)\langle P(a)\rangle,
$$

which means that the $G^{K_{1}, n_{1} ; K_{2}, n_{2} ; \ldots ; K_{k}, n_{k}}$ are invariant tensors of $\tilde{U}_{q}(s u(2))$,

(2) Hermiticity:

$$
\langle P(a)\rangle^{*}=\left\langle P^{*}(a)\right\rangle
$$

for a suitable involution $*$ on $\mathcal{A}$,

(3) Positivity:

$$
\left\langle P(a)^{*} P(a)\right\rangle \geq 0
$$

(4) Symmetry

under permutations of the fields, in a suitable sense discussed below. 
This will be our heuristic "working definition" of a quantum group covariant Euclidean QFT.

In particular, the word "symmetry" in (4) needs some explanation. The main purpose of a symmetrization axiom is that it puts a restriction on the number of degrees of freedom in the model, which in the limit $q \rightarrow 1$ should agree with the undeformed case. More precisely, the amount of information contained in the correlation functions (5.8) should be the same as for $q=1$, i.e. the Poincare series of $\mathcal{A}$ should be the same. This means that the polynomials in the $a^{K, n}$ can be ordered as usual, i.e. they satisfy some kind of "Poincare-Birkhoff-Witt" property. This is what we mean with "symmetry" in (4). In more physical terms, it implies the statistical properties of bosons?.

However, it is far from trivial how to impose such a "symmetry" on tensors which are invariant under a quantum group. Ordinary symmetry is certainly not consistent with covariance under a quantum group. One might be tempted to replace "symmetry" by some kind of invariance under the braid group which is naturally associated to any quantum group. This group is generally much bigger than the group of permutations, however, and such a requirement is qualitatively different and leaves fewer degrees of freedom. The properties (3) and (4) are indeed very nontrivial requirements for a QFT with a quantum group spacetime symmetry, and they are not satisfied in the proposals that have been given up to now, to the knowledge of the authors.

Covariance (1) suggests that the algebra $\mathcal{A}$ generated by the $a^{K, n}$ is a $U_{q}(s u(2))$-module algebra. This implies immediately that $\mathcal{A}$ cannot be commutative, because the coproduct of $U_{q}(s u(2))$ is not cocommutative. The same conclusion can be reached by contemplating the meaning of invariance of an action $S[\Psi]$, which will be clarified below. One could even say that a second quantization is required by consistency. As a further guiding line, the above axioms (1) - (4) should be verified easily in a "free" field theory.

In general, there is no obvious candidate for an associative algebra $\mathcal{A}$ satisfying all these requirements. We will construct a suitable quasiassociative algebra $\mathcal{A}$ as a star-deformation of the algebra of functions on configuration space along the lines of Section 3 , which satisfies these requirements. Our approach is rather general and should be applicable in a more general context, such as for higher-dimensional theories. Quasiassociativity implies that the correlation functions (5.7) make sense only after specifying the order in which the fields should be multiplied (by explicitly putting brackets), however different ways of bracketing are always related by a unitary transformation. Moreover, the correct number of degrees of freedom is guaranteed by construction. We will then define QFT's which satisfy the above requirements using a path integral over the fields $\Psi(x)$, i.e. over the modes $a^{K, n}$. An associative approach will also be presented in Section 5.2, which is essentially equivalent.

\footnotetext{
${ }^{7}$ we do not consider fermions here.
} 


\subsection{Star product approach}

The essential step is as follows. Using the map $\varphi(2.1)$, the coefficients $a^{K, n}$ transform also under the spin $K$ representation of $U(s u(2))$, via $u \tilde{\nabla} a^{K, n}=\varphi^{-1}(u) \tilde{\triangleright}_{q} a^{K, n}$. Hence we can consider the usual commutative algebra $\mathcal{A}^{K}$ of functions on $\mathbb{R}^{2 K+1}$ generated by the $a^{K, n}$, and view it as a left $U(s u(2))$-module algebra $\left(\mathcal{A}^{K}, \cdot, \tilde{\nabla}\right)$. As explained in the Section 3, we can then obtain from it the left $\tilde{U}_{q}(s u(2))$-module algebra $\left(\mathcal{A}^{K}, \star, \tilde{\triangleright}_{q}\right)$, with multiplication $\star$ as defined in (3.2). More generally, we consider the left $\tilde{U}_{q}(s u(2))$-module algebra $\left(\mathcal{A}, \star, \tilde{\triangleright}_{q}\right)$ where $\mathcal{A}=\bigotimes_{K=0}^{N} \mathcal{A}^{K}$. Notice that the twist $\tilde{\mathcal{F}}$ corresponding to the reversed coproduct must be used here, which is simply $\tilde{\mathcal{F}}_{12}=\mathcal{F}_{21}$. The reality issues will be discussed in Section 5.2 .

Invariant actions. Consider the following candidate for an invariant action,

$$
S_{i n t}[\Psi]=\int_{\mathcal{S}_{q, N}^{2}} \Psi(x) \star(\Psi(x) \star \Psi(x)) .
$$

Assuming that the functions on $\mathcal{S}_{q, N}^{2}$ commute with the coefficients, $\left[x_{i}, a^{K, n}\right]=0$, this can be written as

$$
\begin{aligned}
S_{i n t}[\Psi] & =\int_{\mathcal{S}_{q, N}^{2}} \psi_{K, n}(x) \psi_{K^{\prime}, m}(x) \psi_{K^{\prime \prime}, l}(x) a^{K, n} \star\left(a^{K^{\prime}, m} \star a^{K^{\prime \prime}, l}\right) \\
& =I_{K, K^{\prime}, K^{\prime \prime} ; n, m, l}^{(3)} a^{K, n} \star\left(a^{K^{\prime}, m} \star a^{K^{\prime \prime}, l}\right) \quad \in \mathcal{A} .
\end{aligned}
$$

Here $I_{K, K^{\prime}, K^{\prime \prime} ; n, m, l}^{(3)}=\int_{\mathcal{S}_{q, N}^{2}} \psi_{K, n} \psi_{K^{\prime}, m} \psi_{K^{\prime \prime}, l}$ is by construction an invariant tensor of $U_{q}(s u(2))$,

$$
I_{K, K^{\prime}, K^{\prime \prime} ; n, m, l}^{(3)} \pi_{r}^{n}\left(u_{1}\right) \pi_{s}^{m}\left(u_{2}\right) \pi_{t}^{l}\left(u_{3}\right)=\varepsilon_{q}(u) I_{K, K^{\prime}, K^{\prime \prime} ; r, s, t}^{(3)} .
$$

We have omitted the labels on the various representations. Hence $S_{\text {int }}[\Psi]$ is indeed an invariant element of $\mathcal{A}$ :

$$
\begin{aligned}
u \tilde{\triangleright}_{q} S_{i n t}[\Psi] & =I_{K, K^{\prime}, K^{\prime \prime} ; n, m, l}^{(3)} \pi_{r}^{n}\left(\tilde{S} u_{\tilde{1}}\right) \pi_{s}^{m}\left(\tilde{S} u_{\tilde{2}}\right) \pi_{t}^{l}\left(\tilde{S} u_{\tilde{3}}\right) a^{K, r} \star\left(a^{K^{\prime}, s} \star a^{K^{\prime \prime}, t}\right) \\
& =\varepsilon_{q}(u) S_{i n t}[\Psi]
\end{aligned}
$$

\footnotetext{
${ }^{8}$ note that the brackets are actually not necessary here because of 2.20 ). For higher-order terms they are essential, however.
} 
using (5.14), where $u_{\widetilde{1}} \otimes u_{\widetilde{2}} \otimes u_{\widetilde{3}}$ is the 2-fold coproduct of $u \in \tilde{U}_{q}(s u(2))$; notice that the antipode reverses the coproduct. This is the reason for using $\tilde{U}_{q}(\operatorname{su}(2))$.

In general, our actions $S[\Psi]$ will be polynomials in $\mathcal{A}$, and we shall only consider invariant actions,

$$
u \tilde{\triangleright}_{q} S[\Psi]=\varepsilon_{q}(u) S[\Psi] \quad \in \mathcal{A},
$$

for $u \in \tilde{U}_{q}(s u(2))$. It is important to note that by Lemma 3.1, the star product of any such invariant actions is commutative and associative, even though the full algebra of the coefficients $(\mathcal{A}, \star)$ is not. Moreover we only consider actions which are obtained using an integral over $\mathcal{S}_{q, N}^{2}$ as in (5.12), which we shall refer to as "local".

In particular, consider the quadratic action

$$
S_{2}[\Psi]=\int_{\mathcal{S}_{q, N}^{2}} \Psi(x) \star \Psi(x),
$$

which can be rewritten as

$$
\begin{aligned}
S_{2}[\Psi] & =\int_{\mathcal{S}_{q, N}^{2}} \psi_{K, n}(x) \psi_{K, m}(x) a^{K, n} \star a^{K, m}=\sum_{K=0}^{N} g_{n m}^{K} a^{K, n} \star a^{K, m} \\
& =\sum_{K=0}^{N} \tilde{g}_{m n}^{K} a^{K, n} \star a^{K, m} .
\end{aligned}
$$

Here we assumed that the basis $\psi_{K, n}(x)$ is normalized such that

$$
\int_{\mathcal{S}_{q, N}^{2}} \psi_{K, n}(x) \psi_{K^{\prime}, m}(x)=\delta_{K, K^{\prime}} g_{n, m}^{K} .
$$

This action is of course invariant, $u \tilde{\triangleright}_{q} S_{2}[\Psi]=\varepsilon_{q}(u) S_{2}[\Psi]$. Moreover, the invariant quadratic actions agree precisely with the classical ones. Indeed, the most general invariant quadratic action has the form

$$
\begin{aligned}
S_{\text {free }}[\Psi] & =\frac{1}{2} \sum_{K=0}^{N} D_{K} g_{n m}^{K} a^{K, n} \star a^{K, m} \\
& =\frac{1}{2} \sum_{K=0}^{N} D_{K}\left(g_{c}^{K}\right)_{n m} a^{K, n} \cdot a^{K, m}
\end{aligned}
$$

using (3.21), for some $D_{K} \in \mathbb{C}$. This will allow to derive Feynman rules from Gaussian integrals as usual. 
Quantization: path integral. We will define the quantization by a (configuration space) path integral, i.e. some kind of integration over the possible values of the coefficients $a^{K, m}$. This integral should be invariant under $\tilde{U}_{q}(s u(2))$. Following Section 3.1, we consider $\mathcal{A}^{K}$ as the vector space of complex-valued functions on $\mathbb{R}^{2 K+1}$, and use the usual classical integral over $\mathbb{R}^{2 K+1}$. Recall that the algebra structure of $\mathcal{A}^{K}$ does not enter here at all. The same approach was used in [13] to define the quantization of the undeformed fuzzy sphere, and an analogous approach is usually taken on spaces with a star product [26]. Notice that $K$ is an integer, since we do not consider fermionic fields here. Explicitly, let $\int d^{2 K+1} a^{K} f$ be the integral of an element $f \in \mathcal{A}^{K}$ over $\mathbb{R}^{2 K+1}$. It is invariant under the action of $\tilde{U}_{q}(s u(2)$ ) (or equivalently under $U(s u(2)))$ as discussed in Section 3.1:

$$
\int d^{2 K+1} a^{K} u \tilde{\triangleright}_{q} f=\varepsilon_{q}(u) \int d^{2 K+1} a^{K} f .
$$

Now we define

$$
\int \mathcal{D} \Psi f[\Psi]:=\int \prod_{K} d^{2 K+1} a^{K} f[\Psi],
$$

where $f[\Psi] \in \mathcal{A}$ is any integrable function (in the usual sense) of the variables $a^{K, m}$. This will be our path integral, which is by construction invariant under the action $\tilde{\triangleright}_{q}$ of $\tilde{U}_{q}(s u(2))$.

Correlation functions can now be defined as functionals of "bracketed polynomials" $P_{\star}(a)=a^{K_{1}, n_{1}} \star\left(a^{K_{2}, n_{2}} \star\left(\ldots \star a^{K_{l}, n_{l}}\right)\right)$ in the field coefficients by

$$
\left\langle P_{\star}(a)\right\rangle:=\frac{\int \mathcal{D} \Psi e^{-S[\Psi]} P_{\star}(a)}{\int \mathcal{D} \Psi e^{-S[\Psi]}} .
$$

This is natural, because all invariant actions $S[\Psi]$ commute with the generators $a^{K, n}$. Strictly speaking there should be a factor $\frac{1}{\hbar}$ in front of the action, which we shall omit. In fact there are now 3 different "quantization" parameters: $\hbar$ has the usual meaning, while $N$ and $q-q^{-1}$ determines a quantization or deformation of space.

Invariance of the action $S[\Psi] \in \mathcal{A}$ implies that

$$
\left\langle u \tilde{\triangleright}_{q} P_{\star}(a)\right\rangle=\varepsilon_{q}(u)\left\langle P_{\star}(a)\right\rangle,
$$

and therefore

$$
\left\langle P_{\star}(a)\right\rangle=\left\langle\left(P_{0}\right)_{\star}(a)\right\rangle
$$

where $P_{0}$ is the singlet part of the polynomial $P$, as in Lemma 4.4. These are the desired invariance properties, and they would not hold if the $a^{K, n}$ were commuting variables. By construction, the number of independent modes of a polynomial $P_{\star}(a)$ with given degree is 
the same as for $q=1$. One can in fact order them, using quasiassociativity together with the commutation relations $(\overline{3.10})$ which of course also hold under the integral:

$$
\left\langle P_{\star}(a) \star\left(\left(a_{i} \star a_{j}-a_{k} \star a_{l} \tilde{\mathcal{R}}_{i j}^{l k}\right) \star Q_{\star}(a)\right)\right\rangle=0,
$$

for any polynomials $P_{\star}(a), Q_{\star}(a) \in \mathcal{A}$. This can also be verified using the perturbative formula (5.27) below. Therefore the symmetry requirement (4) of Section 5 is satisfied. Moreover, the following cyclic property holds:

$$
\left\langle a_{i} \star P_{\star}(a)\right\rangle=\left\langle P_{\star}(a) \star a_{k}\right\rangle \tilde{D}_{i}^{k}, \quad \tilde{D}_{i}^{k}=\tilde{g}^{k n} \tilde{g}_{i n}
$$

for any $P_{\star}(a)$. This follows using (5.21) and the well-known cyclic property of the $q$-deformed invariant tensor $\tilde{g}_{i j}$.

In general, the use of quasiassociative algebras for QFT is less radical than one might think, and it is consistent with results of [1] on boundary correlation functions in BCFT. Before addressing the issue of reality, we develop some tools to actually calculate such correlation functions in perturbation theory.

Currents and generating functionals. One can now introduce the usual tools of quantum field theory. We introduce (external) currents $J(x)$ by

$$
J(x)=\sum_{K, n} \psi_{K, n}(x) j^{K, n}
$$

where the new generators $j^{K, n}$ are included into the $\tilde{U}_{q}(s u(2))$-module algebra $\mathcal{A}$, again by the twisted product (3.2). We can then define a generating functional

$$
Z[J]=\frac{1}{\mathcal{N}} \int \mathcal{D} \Psi e^{-S[\Psi]+\int \Psi(x) \star J(x)},
$$

which is an element of $\mathcal{A}$ but depends only on the current variables. Here $\mathcal{N}=\int \mathcal{D} \Psi e^{-S[\Psi]}$. Note that

$$
\int \Psi(x) \star J(x)=\int J(x) \star \Psi(x),
$$

which follows e.g. from (5.18). Invariance of the functional integral implies that

$$
u \tilde{\triangleright}_{q} Z[J]=\varepsilon_{q}(u) Z[J]
$$

for any $u \in \tilde{U}_{q}(s u(2))$, provided the actions $S[\Psi]$ are invariant. 
It is now useful to introduce derivatives $\partial_{(j)}^{K, n}$ similar to 3.18$)$, which together with the currents form a twisted (quasiassociative) Heisenberg algebra as explained in the previous section:

$$
\partial_{(j) n}^{K} \star j_{m}^{K^{\prime}}=\delta_{K, K^{\prime}} \tilde{g}_{n m}^{K}+j_{r}^{K^{\prime}} \star \partial_{(j) s}^{K} \tilde{\mathcal{R}}_{n m}^{s r}
$$

By a calculation analogous to (3.24), it follows that

$$
\partial_{(j)}^{K, n}\left(\int \Psi(x) \star J(x)\right)=a^{K, n}+\left(\int \Psi(x) \star J(x)\right) \partial_{(j)}^{K, n} .
$$

Recall that it is not necessary to put a star if one of the factors is a singlet.

This is exactly what we need. We conclude immediately that $\left[\partial_{(j)}^{K, n}, \exp \left(\int \Psi \star J\right)\right]=$ $a^{K, n} \exp \left(\int \Psi \star J\right)$, and by an inductive argument it follows that the correlation functions (5.19) can be written as

$$
\left\langle P_{\star}(a)\right\rangle={ }_{J=0}\left\langle P_{\star}\left(\partial_{(j)}\right) Z[J]\right\rangle_{\partial=0} .
$$

Here ${ }_{J=0}\langle\ldots\rangle_{\partial=0}$ means ordering the derivatives to the right of the currents and then setting $J$ and $\partial_{(j)}$ to zero. The substitution of derivatives into the bracketed polynomial $P_{\star}$ is welldefined, because the algebra of the generators $a$ is the same as the algebra of the derivatives $\partial_{(j)}$.

The usual perturbative expansion can now be obtained easily. Consider a quadratic action of the form

$$
S_{\text {free }}[\Psi]=\int_{\mathcal{S}_{q, N}^{2}} \frac{1}{2} \Psi(x) \star D \Psi(x),
$$

where $D$ is an invariant (e.g. differential) operator on $\mathcal{S}_{q, N}^{2}$, so that $D \Psi(x)=\sum \psi_{K, n}(x) D_{K} a^{K, n}$ with $D_{K} \in \mathbb{C}$. It then follows as usual that

$$
Z_{\text {free }}[J]:=\frac{1}{\mathcal{N}_{\text {free }}} \int \mathcal{D} \Psi e^{-S_{\text {free }}[\Psi]+\int \Psi(x) \star J(x)}=e^{\frac{1}{2} \int J(x) \star D^{-1} J(x)} .
$$

This implies that after writing the full action in the form $S[\Psi]=S_{\text {free }}[\Psi]+S_{\text {int }}[\Psi]$, one has

$$
\begin{aligned}
Z[J] & =\frac{1}{\mathcal{N}} \int \mathcal{D} \Psi e^{-S_{\text {int }}[\Psi]} e^{-S_{\text {free }}[\Psi]+\int \Psi(x) \star J(x)} \\
& \left.=\frac{1}{\mathcal{N}^{\prime}} e^{-S_{\text {int }}\left[\partial_{(j)}\right]} Z_{\text {free }}[J]\right\rangle_{\partial=0} .
\end{aligned}
$$

This is the starting point for a perturbative evaluation. In the next section, we shall cast this into a form which is even more useful, and show that the "vacuum diagrams" cancel as usual. 
Relation with the undeformed case. There is a conceptually simple relation of all the above models which are invariant under $\tilde{U}_{q}(s u(2))$ with models on the undeformed fuzzy sphere which are invariant under $U(s u(2))$, at the expense of "locality". First, note that the space of invariant actions (5.15) is independent of $q$. More explicitly, consider an interaction term of the form (5.13). If we write down explicitly the definition of the $\star$ product of the $a^{K, n}$ variables, then it can be viewed as an interaction term of $a^{K, n}$ variables with a tensor which is invariant under the undeformed $U(s u(2))$, obtained from $I_{K, K^{\prime}, K^{\prime \prime} ; n, m, l}^{(3)}$ by multiplication with representations of $\mathcal{F}$. In the limit $q=1$, this $\mathcal{F}$ becomes trivial. In other words, the above actions can also be viewed as actions on undeformed fuzzy sphere $S_{q=1, N}^{2}$, with interactions which are "nonlocal" in the sense of $S_{q=1, N}^{2}$, i.e. they are given by traces of products of matrices only to the lowest order in $(q-1)$. Upon spelling out the $\star$ product in the correlation functions (5.19) as well, they can be considered as ordinary correlation functions of a slightly nonlocal field theory on $S_{q=1, N}^{2}$, disguised by the transformation $\mathcal{F}$.

In this sense, $q$-deformation simply amounts to some kind of nonlocality of the interactions. A similar interpretation is well-known in the context of field theories on spaces with a Moyal product [26]. The important point is, however, that one can calculate the correlation functions for $q \neq 1$ without using the twist $\mathcal{F}$ explicitly, using only $\hat{R}$-matrices and the coassociators $\tilde{\phi}$, which are much easier to work with. This should make the $q$-deformed point of view useful. It is also possible to generalize these results to other $q$-deformed spaces.

\subsection{Associative approach}

In order to establish the reality properties of the field theories introduced above, it is easier to use an alternative formulation, using the results of Section 1 . The equivalence of the two formulations will follow from Section 4.2. This will also allow to define field operators for second-quantized models in $2+1$ dimensions in Section 6.4.

Consider the left vacuum representations $\left.V_{\mathcal{A}}=\mathcal{A}\right\rangle$ of $\mathcal{A}=\otimes_{K} \mathcal{A}^{K}$ introduced in Section 4, and define the operators?

$$
\hat{a}^{K, n}=\left(\tilde{\mathcal{F}}_{1}^{-1} \triangleright a^{K, n}\right) \tilde{\mathcal{F}}_{2}^{-1} \in U(s u(2)) \ltimes \mathcal{A}
$$

acting on $\mathcal{A}\rangle$. We can then more or less repeat all the constructions of the previous section with $a^{k, n}$ replaced by $\hat{a}^{K, n}$, omitting the $\star$ product. The covariance property (5.6) of the field

$$
\hat{\Psi}(x)=\sum_{K, n} \psi_{K, n}(x) \hat{a}^{K, n}
$$

\footnotetext{
${ }^{9}$ they should not be considered as field operators.
} 
can now be written in the form

$$
\Psi\left[u \triangleright_{q} f\right]=u_{\tilde{1}} \Psi[f] \tilde{S} u_{\tilde{2}} .
$$

Invariant actions can be obtained by contracting the $\hat{a}^{K, n}$ with invariant tensors of $\tilde{U}_{q}(s u(2))$, and satisfy

$$
[u, S[\hat{\Psi}]]=0
$$

for $u \in \tilde{U}_{q}(s u(2))$. For example, any actions of the form

$$
S[\hat{\Psi}]=\int_{\mathcal{S}_{q, N}^{2}} \frac{1}{2} \hat{\Psi}(x) D \hat{\Psi}(x)+\lambda \hat{\Psi}(x) \hat{\Psi}(x) \hat{\Psi}(x)=S_{\text {free }}[\hat{\Psi}]+S_{\text {int }}[\hat{\Psi}] \quad \in U(s u(2)) \ltimes \mathcal{A}
$$

are invariant, where $D$ is defined as before. Using Proposition 1.1, the quadratic invariant actions again coincide with the undeformed ones. In general, higher-order actions are elements of $U(s u(2)) \ltimes \mathcal{A}$ but not of $\mathcal{A}$. Nevertheless as explained in Section 1.2, all such invariant actions $S[\hat{\Psi}]$ are in one-to-one correspondence with invariant actions in the $\star-$ product approach, with brackets as in (4.17). This will be understood from now on.

Consider again the obvious (classical) functional $\int \prod_{K} d^{2 K+1} a^{K}$ on $\mathcal{A}$ (or $V_{\mathcal{A}}$ ) as in the previous section, and recall from Section 4.2 that it extends trivially to a functional on $U(s u(2)) \ltimes \mathcal{A}$, by evaluating $U(s u(2))$ with $\varepsilon$. We will denote this functional by $\int \mathcal{D} \hat{\Psi}$. Define correlation functions of polynomials in the $\hat{a}^{K, n}$ variables as

$$
\langle P(\hat{a})\rangle:=\frac{\int \mathcal{D} \hat{\Psi} e^{-S[\hat{\Psi}]} P(\hat{a})}{\int \mathcal{D} \hat{\Psi} e^{-S[\hat{\Psi}]}}=\left\langle P_{0}(\hat{a})\right\rangle .
$$

Here $P_{0}$ is again the singlet part of the polynomial $P$. Then Lemma 4.4 implies

$$
\langle P(\hat{a})\rangle=\left\langle P_{\star}(a)\right\rangle,
$$

always assuming that the actions $S[\hat{\Psi}]$ are invariant under $U_{q}(s u(2))$. This shows the equivalence with the approach of the previous section. Moreover,

$$
\left\langle P(\hat{a}) \hat{a}_{i} \hat{a}_{j} Q(\hat{a})\right\rangle=\left\langle P(\hat{a}) \hat{a}_{k} \hat{a}_{l} \Re_{i j}^{l k} Q(\hat{a})\right\rangle,
$$

follows from (4.7), or from (5.37) below on the perturbative level.

\footnotetext{
${ }^{10}$ recall that as algebra, there is no difference between $U(s u(2))$ and $\tilde{U}_{q}(s u(2))$.
} 
Currents and generating functionals. We can again extend $\mathcal{A}$ by other variables such as currents

$$
\hat{J}(x)=\sum_{K, n} \psi_{K, n}(x) \hat{j}^{K, n} \quad \in U(s u(2)) \ltimes \mathcal{A},
$$

and consider the generating functional

$$
\left.Z[\hat{J}]=\frac{1}{\mathcal{N}} \int \mathcal{D} \hat{\Psi} e^{-S[\hat{\Psi}]+\int \hat{\Psi}(x) \hat{J}(x)}\right\rangle
$$

with $Z[0]=1$. This is defined as the element of $\mathcal{A}\rangle \cong \mathcal{A}$ obtained after integrating over the $a^{K}$-variables; the result depends on the currents only. The brace $\rangle$ indicates that the explicit $U(s u(2))$ factors in $U(s u(2)) \ltimes \mathcal{A}$ are evaluated by $\varepsilon$. Again, Lemma 4.4 implies that $Z[\hat{J}]$ agrees precisely with the previous definition (5.25).

As explained in Section 4 , one can consider also the twisted derivative operators $\hat{\partial}_{(j)}^{K, n}$, which act on $\mathcal{A}\rangle$. Using Proposition 4.2, we can derive essentially the same formulas as in the previous section, omitting the star product. In particular, (4.15) implies that

$$
\hat{\partial}_{(j)}^{K, n}\left(\int \hat{\Psi}(x) \hat{J}(x)\right)=\hat{a}^{K, n}+\left(\int \hat{\Psi}(x) \hat{J}(x)\right) \hat{\partial}_{(j)}^{K, n}
$$

Since invariant elements of $\mathcal{A}$ are central as was pointed out below (4.4), we obtain as usual

$$
\begin{aligned}
\langle P(\hat{a})\rangle & ={ }_{J=0}\left\langle P\left(\hat{\partial}_{(j)}\right) Z[\hat{J}]\right\rangle_{\partial=0} \\
Z[\hat{J}] & \left.\left.=\frac{1}{\mathcal{N}} \int \mathcal{D} \hat{\Psi} e^{-\left(S_{\text {free }}[\hat{\Psi}]+S_{\text {int }}[\hat{\Psi}]\right)+\int \hat{\Psi}(x) \hat{J}(x)}\right\rangle=\frac{1}{\mathcal{N}^{\prime}} e^{-S_{\text {int }}\left[\hat{\partial}_{(j)}\right]} Z_{\text {free }}[\hat{J}]\right\rangle_{\partial=0} \\
Z_{\text {free }}[\hat{J}] & \left.\left.=\frac{1}{\mathcal{N}_{\text {free }}} \int \mathcal{D} \hat{\Psi} e^{-S_{\text {free }}[\hat{\Psi}]+\int \hat{\Psi}(x) \hat{J}(x)}\right\rangle=e^{\frac{1}{2} \int \hat{J}(x) D^{-1} \hat{J}(x)}\right\rangle .
\end{aligned}
$$

Even though these formulas can be used to calculate correlators perturbatively, there is a form which is more convenient for such calculations. To derive it, observe that (4.13) implies

$$
\hat{\partial}_{(j)}^{K, n} e^{\frac{1}{2} \int \hat{J}(x) D^{-1} \hat{J}(x)}=e^{\frac{1}{2} \int \hat{J}(x) D^{-1} \hat{J}(x)}\left(D_{K}^{-1} \hat{j}^{K, n}+\hat{\partial}_{(j)}^{K, n}\right) ;
$$

one can indeed verify that the algebra of

$$
\hat{b}^{K, n}=D_{K}^{-1} \hat{j}^{K, n}+\hat{\partial}_{(j)}^{K, n}
$$

is the same as the algebra of $\hat{a}^{K, n}$. Therefore (5.37) can be rewritten as

$$
\langle P(\hat{a})\rangle=\frac{1}{\mathcal{N}^{\prime}{ }_{J=0}}\left\langle P\left(\hat{\partial}_{(j)}\right) e^{-S_{i n t}\left[\hat{\partial}_{(j)}\right]} e^{\frac{1}{2} \int \hat{J}(x) D^{-1} \hat{J}(x)}\right\rangle_{\partial=0}
$$




$$
\begin{aligned}
& =\frac{1}{\mathcal{N}^{\prime}}{ }_{J=0}\left\langle e^{\frac{1}{2} \int \hat{J} D^{-1} \hat{J}} P(\hat{b}) e^{-S_{i n t}[\hat{b}]}\right\rangle_{\partial=0} \\
& =\frac{J_{J=0}\left\langle P(\hat{b}) e^{-S_{\text {int }}[\hat{b}]}\right\rangle_{\partial=0}}{{ }_{J=0}\left\langle e^{-S_{\text {int }}[\hat{b}]}\right\rangle_{\partial=0}} .
\end{aligned}
$$

To evaluate this, one reinserts the definition (5.40) of $\hat{b}$ as a sum of derivative operators $\hat{\partial}$ and current generators $\hat{j}$. Each $\hat{\partial}$ must be "contracted" with a $\hat{j}$ to the right of it using the commutation relations (4.14), which gives the inverse propagator $D_{K}^{-1}$, and the result is the sum of all possible complete contractions. This is the analog of Wick's theorem. The contractions can be indicated as usual by pairing up the $\hat{b}$ variables with a line, before actually reordering them. Then each contribution can be reconstructed uniquely from a given complete contraction; this could be stated in terms of Feynman rules.

One can also show that the denominator exactly cancels the "vacuum bubbles" in the numerator, as usual. Indeed, consider any given complete contraction of a term

$$
\hat{b} \ldots \hat{b} \frac{1}{n !}\left(S_{\text {int }}[\hat{b}]\right)^{n} .
$$

Mark the set of vertices which are connected (via a series of contractions) to some of the explicit $\hat{b}$ generators on the left with blue, and the others with red. Then 2 neighboring red and blue vertices can be interchanged keeping the given contractions, without changing the result. This is because only the homogeneous part of the commutation relations (4.14) applies, and all vertices are singlets (cp. Lemma 4.4). Therefore the red vertices can be moved to the right of the blue ones, and their contractions are completely disentangled. Then the usual combinatorics yields

$$
\langle P(\hat{a})\rangle={ }_{J=0}\left\langle P(\hat{b}) e^{-S_{\text {int }}[\hat{b}]}\right\rangle_{\partial=0, \text { no vac }}
$$

in self-explanatory notation. Of course this also holds in the quasiassociative version, but the derivation is perhaps less transparent.

In general, it is not easy to evaluate these expressions explicitly, because of the coassociators. However the lowest-order corrections $o(h)$ where $q=e^{h}$ are easy to obtain, using the fact that $\tilde{\phi}=\mathbf{1}+o\left(h^{2}\right)$ for minimal twists (2.13). If we write

$$
R_{12}=\mathbf{1}+h r_{12}+o\left(h^{2}\right),
$$

then

$$
\tilde{R}_{12}=R_{12}{\sqrt{R_{21} R_{12}}}^{-1}=\mathbf{1}+\frac{h}{2}\left(r_{12}-r_{21}\right)+o\left(h^{2}\right)
$$

which allows to find the leading $o(h)$ corrections to the undeformed correlation functions explicitly.

\footnotetext{
${ }^{11}$ associativity helps here.
} 
Reality structure. One advantage of this formalism is that the reality structure is naturally induced from the Hilbert space $V_{\mathcal{A}}$, as explained in Section 4.1. Using Proposition 4.3 and noting that the $a^{K, m}$ are in the contragredient representation of $U(s u(2))$, it follows that

$$
\left(\hat{a}^{K, n}\right)^{*}=\tilde{g}_{n m}^{K} \hat{a}^{K, m} .
$$

We shall assume that all the actions are real,

$$
S[\hat{\Psi}]^{*}=S[\hat{\Psi}]
$$

this will be verified in the examples below. Moreover, the classical integral defines a real functional on $U(s u(2)) \ltimes \mathcal{A}$. Hence we conclude that the correlation functions satisfy

$$
\langle P(\hat{a})\rangle^{*}=\left\langle P(\hat{a})^{*}\right\rangle .
$$

One can also show that

$$
\psi_{I, i}(x)^{*}=g_{i j}^{I} \psi_{I, j}(x),
$$

where $g_{i j}^{I}$ is normalized such that $g_{i j}^{I}=\left(g^{I}\right)^{i j}$. Therefore

$$
\hat{\Psi}(x)^{*}=\hat{\Psi}(x),
$$

using (5.4). This is useful to establish the reality of actions. Of course, one could also consider complex scalar fields. Finally, the correlation functions satisfy the positivity property

$$
\left\langle P(\hat{a})^{*} P(\hat{a})\right\rangle \geq 0
$$

provided the actions are real. This is a simple consequence of the fact that $P(\hat{a})^{*} P(\hat{a})$ is a positive operator acting on the left vacuum representation, together with the positivity of the functional integral. It is one of the main merits of the present approach.

\section{$6 \quad$ Examples}

\subsection{The free scalar field}

Consider the action

$$
S_{\text {free }}[\Psi]=-\int_{\mathcal{S}_{q, N}^{2}} \frac{1}{2} \hat{\Psi}(x) \star \Delta \Psi(x) .
$$


Here the Laplacian was defined in [12] using a differential calculus as $\Delta=*_{H} d *_{H} d$, and satisfies $[2$

$$
\Delta \psi_{K, n}(x)=\frac{1}{R^{2}}[K]_{q}[K+1]_{q} \psi_{K, n}(x) \equiv D_{K} \psi_{K, n}(x),
$$

where $[K]_{q}=\frac{q^{K}-q^{-K}}{q-q^{-1}}$. The basis $\psi_{K, n}(x)$ is normalized as in (5.17). The action is real by (5.46), and can be rewritten as

$$
S_{\text {free }}[\hat{\Psi}]=-\sum_{K, n} \frac{1}{2} D_{K} \tilde{g}_{n m}^{K} \hat{a}^{K, m} \hat{a}^{K, n}=-\sum_{K, n} \frac{1}{2} D_{K}\left(\tilde{g}^{K}\right)^{m n} \hat{a}_{K, m} \hat{a}_{K, n},
$$

using (5.5). As a first exercise, we calculate the 2-point functions. From (5.37) and (5.38), one finds

$$
\begin{aligned}
\left\langle\hat{a}_{n}^{K} \hat{a}_{n^{\prime}}^{K^{\prime}}\right\rangle & ={ }_{J=0}\left\langle\hat{\partial}_{n}^{K} \hat{\partial}_{n^{\prime}}^{K^{\prime}} Z_{\text {free }}[\hat{J}]\right\rangle_{\partial=0} \\
& ={ }_{J=0}\left\langle\frac{1}{2} \hat{\partial}_{n}^{K} \hat{\partial}_{n^{\prime}}^{K^{\prime}}\left(\sum\left(\tilde{g}^{K}\right)^{r s} \hat{j}_{r}^{K} D_{K}^{-1} \hat{j}_{s}^{K}\right)\right\rangle_{\partial=0} \\
& =D_{K}^{-1}{ }_{J=0}\left\langle\hat{\partial}_{n}^{K} \hat{j}_{n^{\prime}}^{K^{\prime}}\right\rangle_{\partial=0}=D_{K}^{-1} \delta^{K K^{\prime}} \tilde{g}_{n n^{\prime}}^{K}
\end{aligned}
$$

where (4.13) was used in the last line. This result is as expected, and it could also be obtained by using explicitly the definition of the twisted operators $\hat{a}^{K}$.

The calculation of the 4-point functions is more complicated, since it involves the coassociator. To simplify the notation, we consider the (most complicated) case where all generators $a^{K}$ have the same spin $K$, which will be suppressed. The result for the other cases can then be deduced easily. We also omit the prescriptions $(\partial=0)$ etc. Using first the associative formalism, (5.41) yields

$$
\begin{aligned}
\left\langle\hat{a}_{n} \hat{a}_{m} \hat{a}_{k} \hat{a}_{l}\right\rangle & =\left\langle\left(D^{-1} \hat{j}_{n}+\hat{\partial}_{n}\right)\left(D^{-1} \hat{j}_{m}+\hat{\partial}_{m}\right)\left(D^{-1} \hat{j}_{k}+\hat{\partial}_{k}\right)\left(D^{-1} \hat{j}_{l}+\hat{\partial}_{l}\right)\right\rangle \\
& =\left\langle\hat{\partial}_{n}\left(D^{-1} \hat{j}_{m}+\hat{\partial}_{m}\right)\left(D^{-1} \hat{j}_{k}+\hat{\partial}_{k}\right) D^{-1} \hat{j}_{l}\right\rangle \\
& =D^{-2}\left\langle\hat{\partial}_{n} \hat{j}_{m} \tilde{g}_{k l}+\hat{\partial}_{n} \hat{\partial}_{m} \hat{j}_{k} \hat{j}_{l}\right\rangle \\
& =D^{-2}\left\langle\tilde{g}_{n m} \tilde{g}_{k l}+\hat{\partial}_{n} \hat{\partial}_{m} \hat{j}_{k} \hat{j}_{l}\right\rangle
\end{aligned}
$$

To evaluate this, consider

$$
\begin{aligned}
\left\langle\hat{\partial}_{n} \hat{\partial}_{m} \hat{j}_{k} \hat{j}_{l}\right\rangle & =\left\langle\hat{\partial}_{n}\left(\tilde{g}_{m k}+\hat{j}_{a} \hat{\partial}_{b} \Re_{m k}^{b a}\right) \hat{j}_{l}\right\rangle \\
& =\tilde{g}_{m k} \tilde{g}_{n l}+\left\langle\hat{\partial}_{n} \hat{j}_{a} \hat{\partial}_{b} \hat{j}_{s} \pi_{l}^{s}\left(\Re_{m k}^{b a}\right)\right\rangle \\
& =\tilde{g}_{m k} \tilde{g}_{n l}+\tilde{g}_{n a} \tilde{g}_{b s}\left(\tilde{\phi}_{213} \tilde{R}_{12} \tilde{\phi}^{-1}\right)_{m k l}^{b a s}
\end{aligned}
$$

\footnotetext{
12 it is rescaled from the one in $[12$ so that its eigenvalues are independent of $N$.
} 
Collecting the result, we recognize the structure of Wick contractions which are given by the invariant tensor for neighboring indices, but involve the $\tilde{R}$-matrix and the coassociator $\tilde{\phi}$ for "non-planar" diagrams.

To illustrate the quasiassociative approach, we calculate the same 4-point function using the $\star$ product. Then

$$
\begin{aligned}
\left\langle a_{n} \star\left(a_{m} \star\left(a_{k} \star a_{l}\right)\right)\right\rangle & =\left\langle\partial_{n} \star\left(\left(D^{-1} j_{m}+\partial_{m}\right) \star\left(\left(D^{-1} j_{k}+\partial_{k}\right) \star D^{-1} j_{l}\right)\right)\right\rangle \\
& =D^{-2} \tilde{g}_{n m} \tilde{g}_{k l}+D^{-2}\left\langle\partial_{n} \star\left(\partial_{m} \star\left(j_{k} \star j_{l}\right)\right)\right\rangle
\end{aligned}
$$

using an obvious analog of (5.41). Now

$$
\begin{aligned}
\left\langle\partial_{n} \star\left(\partial_{m} \star\left(j_{k} \star j_{l}\right)\right)\right\rangle & =\left\langle\partial_{n} \star\left(\left(\partial_{m^{\prime}} \star j_{k^{\prime}}\right) \star j_{l^{\prime}}\right)\right\rangle\left(\tilde{\phi}^{-1}\right)_{m k l}^{m^{\prime} k^{\prime} l^{\prime}} \\
& =\tilde{g}_{n l^{\prime}} \tilde{g}_{m^{\prime} k^{\prime}}\left(\tilde{\phi}^{-1}\right)_{m k l}^{m^{\prime} k^{\prime} l^{\prime}}+\left\langle\partial_{n} \star\left(\left(j_{m^{\prime \prime}} \star \partial_{k^{\prime \prime}} \tilde{R}_{m^{\prime} k^{\prime}}^{k^{\prime \prime} m^{\prime \prime}}\right) \star j_{l^{\prime}}\right)\right\rangle\left(\tilde{\phi}^{-1}\right)_{m k l}^{m^{\prime} k^{\prime} l^{\prime}} \\
& =\tilde{g}_{n l} \tilde{g}_{m k}+\left\langle\partial_{n} \star\left(j_{m^{\prime}} \star\left(\partial_{k^{\prime}} \star j_{l^{\prime}}\right)\right\rangle\left(\tilde{\phi}_{213} \tilde{R}_{12} \tilde{\phi}^{-1}\right)_{m k l}^{k^{\prime} m^{\prime} l^{\prime}}\right. \\
& =\tilde{g}_{n l} \tilde{g}_{m k}+\tilde{g}_{n m^{\prime}} \tilde{g}_{k^{\prime} l^{\prime}}\left(\tilde{\phi}_{213} \tilde{R}_{12} \tilde{\phi}^{-1}\right)_{m k l}^{k^{\prime} m^{\prime} l^{\prime}}
\end{aligned}
$$

in agreement with our previous calculation; here the identity (7.4) was used. As pointed out before, the corrections to order $o(h)$ can now be obtained easily.

\subsection{Remarks on $N \rightarrow \infty$ and $\phi^{4}$ theory.}

The above correlators for the free theory are independent of $N$, as long as the spin of the modes is smaller than $N$. Therefore one can define the limit $N \rightarrow \infty$ in a straightforward way, keeping $R$ constant. In this limit, the algebra of functions on the $q$-deformed fuzzy sphere becomes

$$
\varepsilon_{k}^{i j} x_{i} x_{j}=R\left(q-q^{-1}\right) x_{k}, \quad g^{i j} x_{i} x_{j}=R^{2},
$$

which defines $S_{q, N=\infty}^{2}$. It has a unique faithful (infinite-dimensional) Hilbert space representation [24].

In an interacting theory, the existence of the limit $N \rightarrow \infty$ is of course a highly nontrivial question. Consider for example the $\phi^{4}$ model, with action

$$
S[\Psi]=\int_{\mathcal{S}_{q, N}^{2}} \frac{1}{2} \hat{\Psi}(x) \Delta \hat{\Psi}(x)+\frac{1}{2} m^{2} \hat{\Psi}(x)^{2}+\lambda \hat{\Psi}(x)^{4}=S_{\text {free }}+S_{\text {int }}
$$

which is real, using (5.46). We want to study the first-order corrections in $\lambda$ to the $2-$ point function $\left\langle\hat{a}_{i}^{K} \hat{a}_{j}^{K}\right\rangle$ using (5.42):

$$
\left\langle\hat{a}_{i}^{K} \hat{a}_{j}^{K}\right\rangle={ }_{J=0}\left\langle\hat{b}_{i}^{K} \hat{b}_{j}^{K}\left(1-\lambda \int_{\mathcal{S}_{q, N}^{2}} \psi^{I, k}(x) \psi^{J, l}(x) \psi^{L, m}(x) \psi^{M, n}(x) \hat{b}_{k}^{I} \hat{b}_{l}^{J} \hat{b}_{m}^{L} \hat{b}_{n}^{M}\right)\right\rangle_{\partial=0, \text { no vac }}
$$


We only consider the "leading" planar tadpole diagram. It is given by any contraction of the $\hat{b}_{i}^{K}$ and $\hat{b}_{j}^{K}$ with $\hat{b}$ 's in the interaction term, which does not involve "crossings". All of these contributions are the same, hence we assume that $j$ is contracted with $k$ and $i$ with $l$. Then $\hat{b}_{m}^{L}$ is contracted with $\hat{b}_{n}^{M}$, which gives $D_{L}^{-1} \tilde{g}_{m n}^{L} \delta^{L M}$. Now $\psi^{L, m}(x) \psi^{L, n}(x) \tilde{g}_{m n}^{L} \in \mathcal{S}_{q, N}^{2}$ is invariant under $U_{q}(s u(2))$ and therefore proportional to the constant function. The numerical factor can be obtained from (5.17):

$$
\int \psi^{L, m}(x) \psi^{L, n}(x) \tilde{g}_{m n}^{L}=\tilde{g}_{L}^{m n} \tilde{g}_{m n}^{L}=[2 L+1]_{q}={ }_{q} \operatorname{dim}\left(V^{L}\right) .
$$

Here $V^{L}$ denotes the spin $L$ representation of $U_{q}(s u(2))$. Using $\int 1=4 \pi R^{2}$, the contribution to $\left\langle\hat{a}_{i}^{K} \hat{a}_{j}^{K}\right\rangle$ is

$$
\tilde{g}_{i l}^{K} \tilde{g}_{j k}^{K} \lambda \int \psi^{K, k}(x) \psi^{K, l}(x) \sum_{L=0}^{N} D_{L}^{-1} \frac{1}{4 \pi R^{2}}[2 L+1]_{q}=\tilde{g}_{i j}^{K} \frac{\lambda}{4 \pi} \sum_{L=0}^{N} \frac{[2 L+1]_{q}}{[L]_{q}[L+1]_{q}+m^{2} R^{2}},
$$

up to combinatorial factors of order 1 . Unfortunately this diverges linearly in $N$ for $N \rightarrow \infty$, whenever $q \neq 1$. This is worse that for $q=1$, where the divergence is only logarithmic. This is in contrast to a result of [23], which is however in the context of a different concept of (braided) quantum field theory which does not satisfy our requirements in Section 5, and hence is not a "smooth deformation" of ordinary QFT. The contributions from the "nonplanar" tadpole diagrams are expected to be smaller, because the coassociator $\tilde{\phi}$ as well as $\tilde{R}$ are unitary. At least for scalar field theories, this behavior could be improved by choosing another Laplacian such as $\frac{v-v^{-1}}{q-q^{-1}}$ which has eigenvalues $[2 L(L+1)]_{q}$, where $v$ is the Drinfel'd Casimir (3.16). Then all diagrams are convergent as $N \rightarrow \infty$. Finally, the case $q$ being a root of unity is much more subtle, and we postpone it for future work.

\subsection{Gauge fields}

The quantization of gauge fields $\mathcal{S}_{q, N}^{2}$ is less clear at present, and we will briefly indicate 2 possibilities. Gauge fields were introduced in [12] as one-forms $B \in \Omega_{q, N}^{1}$. Here $\Omega_{q, N}^{1}$ is the subspace of one-forms in the $U_{q}(s u(2))$-module algebra of differential forms on $\mathcal{S}_{q, N}^{2}$. It turns out that there is a basis of 3 independent one-forms $\theta^{a}$ which commute with all functions on $\mathcal{S}_{q, N}^{2}$. It is then natural to expand the gauge fields in that basis,

$$
B=\sum B_{a} \theta^{a}
$$

The fact that there are 3 independent one-forms means that one component is essentially radial and should be considered as a scalar field on the sphere; however, it is impossible to 
find a (covariant) calculus with "tangential" forms only. Therefore gauge theory on $\mathcal{S}_{q, N}^{2}$ as presented here is somewhat different from the conventional picture, but may nevertheless be very interesting physically [2].

Actions for gauge theories are expressions in $B$ which involve no explicit derivative terms. Examples are

$$
S_{3}=\int B^{3}, \quad S_{2}=\int B *_{H} B, \quad S_{4}=\int B^{2} *_{H} B^{2},
$$

where $*_{H}$ is the Hodge star operator. The curvature can be defined as $F=B^{2}-*_{H} B$. The meaning of the field $B$ becomes more obvious if it is written in the form

$$
B=\Theta+A
$$

where $\Theta \in \Omega_{q, N}^{1}$ is the "Dirac-operator". While $B$ and $\Theta$ become singular in the limit $N \rightarrow \infty, A$ remains well-defined. In these variables, a more standard form of the actions is recovered, including Yang-Mills

$$
S_{Y M}:=\int F *_{H} F=\int\left(d A+A^{2}\right) *_{H}\left(d A+A^{2}\right)
$$

and Chern-Simons

$$
S_{C S}:=\frac{1}{3} \int B^{3}-\frac{1}{2} \int B *_{H} B=- \text { const }+\frac{1}{2} \int A d A+\frac{2}{3} A^{3}
$$

terms. For further details we refer to [12].

Even though these actions (in particular the prescription "no explicit derivatives") are very convincing and have the correct limit at $q=1$, the precise meaning of gauge invariance is not clear. In the case $q=1$, gauge transformations have the form $B_{a} \rightarrow U^{-1} B_{a} U$ for any unitary matrix $U$, and actions of the above type are invariant. For $q \neq 1$, the integral is a quantum trace which contains an explicit "weight factor" $q^{-H}$, breaking this symmetry. There is however another symmetry of the above actions where $U_{q}(s u(2))$ acts on the gauge fields $B_{a}$ as 12

$$
B_{a} \rightarrow u_{1} B_{a} S u_{2}
$$

or equivalently $B \rightarrow u_{1} B S u_{2}$. This can be interpreted as a gauge transformation, leaving the actions invariant for any $u \in U_{q}(s u(2))$ with $\varepsilon_{q}(u)=1$, and it is distinct from the rotations of $B$. There is no obvious extension to a deformed $U(s u(N))$ invariance, however. There is yet another $\tilde{U}_{q}(s u(2))$ symmetry, rotating the frames $\theta^{a}$ only, i.e. mixing the components $B_{a}$. The rotation of the field $B$ is rather complicated if expressed in terms of the $B_{a}$, however.

The significance of all these different symmetries is not clear, and we are not able to preserve them simultaneously at the quantum level. We will therefore indicate two possible quantization schemes, leaving different symmetries manifest. 
1) Quantization respecting rotation-invariance. First, we want to preserve the $U_{q}(s u(2))$ symmetry corresponding to rotations of the one-forms $\Omega_{q, N}^{1}$, which is underlies their algebraic properties [12]. We shall moreover impose the constraint

$$
d *_{H} B=0,
$$

which can be interpreted as gauge fixing. It is invariant under rotations, and removes precisely the null-modes in the Yang-Mills and Chern-Simons terms. We expand the field $B$ into irreducible representations under this action of $U_{q}(s u(2))$ :

$$
B=\sum_{K, n ; \alpha} \Xi_{K, n}^{\alpha}(x) b_{\alpha}^{K, n}
$$

Here $\Xi_{K, n}^{\alpha}(x) \in \Omega_{q, N}^{1}$ are one-forms which are spin $K$ representation of $U_{q}(s u(2)$ ) ("vector spherical harmonics"). The multiplicity is now generically 2 because of the constraint, labeled by $\alpha$.

To quantize this, we can use the same methods as in Section 5. One can either define a * product of the coefficients $b_{\alpha}^{K, n}$ as discussed there, or introduce the operators $\hat{b}_{\alpha}^{K, n}$ acting on a left vacuum representation. Choosing the star product approach to be specific, one can then define correlation functions as

$$
\left\langle P_{\star}(b)\right\rangle=\frac{1}{\mathcal{N}} \int \mathcal{D} B e^{-S[B]} P_{\star}(b)
$$

where $\mathcal{D} B$ is the integral over all $b_{\alpha}^{K, n}$, write down generating functions etc. This approach has the merit that the remarkable solution $B=\Theta$ of the equation $F=0$ in [12] survives the quantization, because the corresponding mode is a singlet (so that $\hat{b}_{\alpha}^{0,0}=b_{\alpha}^{0,0}$ is undeformed). Incidentally, observe that the bracketings $\int(B B) *_{H}(B B)$ and $\int B\left(B *_{H}(B B)\right)$ in the starproduct approach are equivalent, because of (2.13).

2) Quantization respecting "gauge invariance". First, notice that there is no need for gauge fixing before quantization even for $q=1$, since the group of gauge transformations is compact. To preserve the symmetry (6.8) as well as the rotation of the $\theta^{a}$, we expand $B$ into irreducible representations under these 2 symmetries $U_{q}(s u(2))$ and $\tilde{U}_{q}(s u(2))$ :

$$
B=\sum_{K, n ; a} \psi_{K, n}(x) \theta^{a} \beta_{a}^{K, n}
$$

Now $\beta_{a}^{K, n}$ is a spin $K$ representation of $\tilde{U}_{q}(s u(2))$ and a spin 1 representation of $U_{q}(s u(2))$. These are independent and commuting symmetries, hence the quantization will involve their 
respective Drinfel'd twists $\tilde{\mathcal{F}}$ and $\mathcal{F}$. In the associative approach of Section 5 we would then introduce

$$
\hat{\beta}_{a}^{K, n}=\beta_{a^{\prime}}^{K, n^{\prime}} \pi_{a}^{a^{\prime}}\left(\mathcal{F}_{1}^{-1}\right) \pi_{n^{\prime}}^{n}\left(\tilde{S} \tilde{\mathcal{F}}_{1}^{-1}\right) \mathcal{F}_{2}^{-1} \tilde{\mathcal{F}}_{2}^{-1} \in(\tilde{U}(s u(2)) \otimes U(s u(2))) \ltimes \mathcal{A} .
$$

To avoid confusion, we have used an explicit matrix notation here. The rest is formally as before, and will be omitted. One drawback of this approach is that the above-mentioned solution $B=\Theta$ is somewhat obscured now: the corresponding mode is part of $\beta_{a}^{1, n}$, but not easily identified. Moreover, "overall" rotation invariance is not manifest in this quantization.

\subsection{QFT in $2_{q}+1$ dimensions, Fock space}

So far, we considered 2-dimensional $q$-deformed Euclidean field theory. In this section, we will add an extra (commutative) time and define a 2+1-dimensional scalar quantum field theory on $\mathcal{S}_{q, N}^{2}$ with manifest $\tilde{U}_{q}(s u(2)) \times \mathbb{R}$ symmetry, where $\mathbb{R}$ corresponds to time translations. This will be done using an operator approach, with $q$-deformed creation and anihilation operators acting on a Fock space. The purpose is mainly to elucidate the meaning of the Drinfel'd twists as "dressing transformations".

We consider real scalar field operators of the form

$$
\hat{\Psi}(x, t)=\sum_{K, n} \psi^{K, n}(x) \hat{a}_{K, n}(t)+\psi^{K, n}(x)^{*} \hat{a}_{K, n}^{+}(t)
$$

where

$$
a_{K, n}^{(+)}(t)=U^{-1}(t) a_{K, n}^{(+)}(0) U(t)
$$

for some unitary time-evolution operator $U(t)=e^{-i H t / \hbar}$; we will again put $\hbar=1$. The Hamilton-operator $H$ acts on some Hilbert space $\mathcal{H}$. We will assume that $H$ is invariant under rotations,

$$
[H, u]=0
$$

where $u \in U(s u(2))$ is an operator acting on $\mathcal{H}$; recall that as (operator) algebra, $\tilde{U}_{q}(s u(2))$ is the same as $U(s u(2))$. Rather than attempting some kind of quantization procedure, we shall assume that

$$
\hat{a}_{K, n}^{(+)}(t)=\tilde{\mathcal{F}}_{1}^{-1} \triangleright a_{K, n}^{(+)}(t) \tilde{\mathcal{F}}_{2}^{-1}=a_{K, m}^{(+)}(t) \pi_{n}^{m}\left(\tilde{\mathcal{F}}_{1}^{-1}\right) \tilde{\mathcal{F}}_{2}^{-1}
$$

as in (4.1), where $a_{K, n}^{(+)}=a_{K, n}^{(+)}(0)$ are ordinary creation-and anihilation operators generating a oscillator algebra $\mathcal{A}$,

$$
\begin{aligned}
& {\left[a_{K, n}, a_{K^{\prime}, n^{\prime}}^{+}\right]=\delta_{K K^{\prime}}\left(g_{c}\right)_{n n^{\prime}},} \\
& {\left[a_{K, n}, a_{K^{\prime}, n^{\prime}}\right]=\left[a_{K, n}^{+}, a_{K^{\prime}, n^{\prime}}^{+}\right]=0}
\end{aligned}
$$


and act on the usual Fock space ${ }^{13}$

$$
\mathcal{H}=\oplus\left(a_{K, n}^{+} \ldots a_{K^{\prime}, n^{\prime}}^{+}|0\rangle\right) .
$$

$\mathcal{H}$ is in fact a representation of $U(s u(2)) \ltimes \mathcal{A}$, and the explicit $U(s u(2))$-terms in (6.14) are now understood as operators acting on $\mathcal{H}$. Hence the $\hat{a}_{K, n}^{(+)}(t)$ are some kind of dressed creation-and anihilation operators, whose equal-time commutation relations follow from (4.7), (4.14):

$$
\begin{aligned}
\hat{a}_{K, n} \hat{a}_{K^{\prime}, n^{\prime}}^{+} & =\delta_{K, K^{\prime}} g_{n n^{\prime}}+\hat{a}_{K^{\prime}, l^{\prime}}^{+} \hat{a}_{K, l} \mathfrak{R}_{n n^{\prime}}^{l l^{\prime}}, \\
\hat{a}_{K, n}^{+} \hat{a}_{K^{\prime}, n^{\prime}}^{+} & =\hat{a}_{K^{\prime}, l^{\prime}}^{+} \hat{a}_{K, l}^{+} \mathfrak{R}_{n n^{\prime}}^{l l^{\prime}}, \\
\hat{a}_{K, n} \hat{a}_{K^{\prime}, n^{\prime}} & =\hat{a}_{K^{\prime}, l^{\prime}} \hat{a}_{K, l} \mathfrak{R}_{n n^{\prime}}^{l l^{\prime}}
\end{aligned}
$$

where $\hat{a}_{K, n}^{(+)}=\hat{a}_{K, n}^{(+)}(0)$. The Fock space (6.15) can equivalently be written as

$$
\mathcal{H}=\oplus \hat{a}^{+K, n} \ldots \hat{a}^{+K^{\prime}, n^{\prime}}|0\rangle
$$

Here the main point of our construction of a quantum group covariant field theory is most obvious, namely that a symmetrization postulate has been implemented which restricts the number of states in the Hilbert space as in the undeformed case. This is the meaning of the postulate (4) in the introductory discussion of Section 5. One could even exhibit a (trivial) action of the symmetric group $S_{n}$ on the $n$-particle space, using the unitary transformation induced by the Drinfel'd twist $\mathcal{F}$, as in [11]. Moreover, using (5.45) and an analog of (5.43) it follows that

$$
\hat{\Psi}(x, t)^{*}=\hat{\Psi}(x, t) .
$$

One can also derive the usual formulas for time-dependent perturbation theory, if we assume that the Hamilton operator has the form

$$
H=H_{\text {free }}+V
$$

where

$$
H_{\text {free }}=\sum_{K=0}^{N} D_{K}\left(\tilde{g}^{K}\right)^{n m} \hat{a}_{K, n}^{+} \hat{a}_{K, m}=\sum_{K=0}^{N} D_{K}\left(g_{c}^{K}\right)^{n m} a_{K, n}^{+} a_{K, m},
$$

and $V$ may have the form

$$
V=\int_{\mathcal{S}_{q, N}^{2}} \hat{\Psi}(x) \hat{\Psi}(x) \ldots \hat{\Psi}(x) .
$$

\footnotetext{
${ }^{13}$ note that this is the same as the "left vacuum representation" of the subalgebra generated by the $a_{K, n}^{+}$, in the notation of Section 4 .
} 
Using (4.15), one can see that

$$
\left[H_{\text {free }}, \hat{a}_{K, l}^{+}\right]=D_{K} \hat{a}_{K, l}^{+}
$$

and similarly for $\hat{a}_{K, l}$. Therefore the eigenvectors of $H_{\text {free }}$ have the form $\hat{a}^{+K, n} \ldots \hat{a}^{+K^{\prime}, n^{\prime}}|0\rangle$ with eigenvalues $\left(D_{K}+\ldots+D_{K^{\prime}}\right) \in \mathbb{R}$, and if $V=0$, then the time evolution is given as usual by

$$
\hat{a}_{K, n}^{+}(t)=e^{-i D_{K} t / \hbar} \hat{a}_{K, n}^{+}, \quad \hat{a}_{K, n}(t)=e^{i D_{K} t / \hbar} \hat{a}_{K, n} .
$$

One can then go to the interaction picture if $V \neq 0$ and derive the usual formula involving time-ordered products. However one must now keep the time-ordering explicit, and there seems to be no nice formula for contractions of time-ordered products. We shall not pursue this any further here.

The main point here is that the above definitions are entirely within the framework of ordinary quantum mechanics, with a smooth limit $q \rightarrow 1$ where the standard quantum field theory on the fuzzy sphere is recovered. Again, one could also consider the limit $N \rightarrow \infty$ while keeping $q$ constant. The existence of this limit is far from trivial. Moreover there is nothing special about the space $\mathcal{S}_{q, N}^{2}$ as opposed to other, perhaps higher-dimensional $q-$ deformed spaces, except the technical simplifications because of the finite number of modes. This shows that there is no obstacle in principle for studying deformations of quantum field theory on such spaces.

Acknowledgements: We would like to thank C.-S. Chu, G. Fiore and J. Pawelczyk for useful and stimulating discussions, and J. Wess for support and hospitality at the MaxPlanck Institut für Physik in München. H. S. and J. M. are grateful for useful visits at the Erwin-Schrödinger Institut in Vienna, and H. G and J. M. thank K. Sibold and E. Zeidler for hospitality at the University of Leipzig resp. the MPI in Leipzig. H. S. also thanks the DFG for a fellowship, as well as D. Schiff for hospitality at the Laboratoire de Physique Théorique in Orsay.

\section{Appendix A: some proofs}

Proof of Proposition 2.1: Assume that $\mathcal{F}$ is minimal, so that (2.13) holds. We must show that it can be chosen such that $\mathcal{F}$ is unitary as well. Define

$$
A:=\mathcal{F}_{23}(1 \otimes \Delta) \mathcal{F}, \quad B:=\mathcal{F}_{12}(\Delta \otimes 1) \mathcal{F},
$$

so that $\phi=B^{-1} A$. From (2.13) it follows that $(* \otimes * \otimes *) \phi=\phi^{-1}$, hence $A A^{*}=B B^{*}$, and more generally

$$
f\left(A A^{*}\right)=f\left(B B^{*}\right)
$$


for functions $f$ which are defined by a power series. This also implies that

$$
A f\left(A^{*} A\right) A^{*}=B f\left(B^{*} B\right) B^{*}
$$

for any such $f$, hence

$$
\phi f\left(A^{*} A\right)=f\left(B^{*} B\right) \phi^{*-1}=f\left(B^{*} B\right) \phi .
$$

In particular we can choose $f(x)=\sqrt{x}$ which makes sense because of (2.3), and obtain

$$
{\sqrt{B^{*} B}}^{-1} \phi \sqrt{A^{*} A}=\phi \text {. }
$$

On the other hand, the element $T:=((* \otimes *) \mathcal{F}) \mathcal{F}$ commutes with $\Delta(u)$ because $(* \otimes *) \Delta_{q}(u)=$ $\Delta_{q}\left(u^{*}\right)$, and so does $\sqrt{T}$, which is well-defined in $U(s u(2))[[h]]$ since $\mathcal{F}=1+o(h)$. Moreover, $T$ is symmetric, noting that

$$
(* \otimes *)\left(\mathcal{F}_{21} \mathcal{F}^{-1}\right)=\mathcal{F} \mathcal{F}_{21}^{-1}
$$

which follows from the well-known relation $(* \otimes *) \mathcal{R}=\mathcal{R}_{21}$ for $q \in \mathbb{R}$. Therefore $T$ is an admissible gauge transformation, and $\mathcal{F}^{\prime}:=\mathcal{F} \sqrt{T}^{-1}$ is easily seen to be unitary (this argument is due to [16]). In particular, since $\mathcal{F}^{*} \mathcal{F}$ commutes with $\Delta(u)$, it follows that $A^{*} A=$ $\left(\mathcal{F}_{23}^{*} \mathcal{F}_{23}\right)(1 \otimes \Delta)\left(\mathcal{F}^{*} \mathcal{F}\right)$ and $B^{*} B=\left(\mathcal{F}_{12}^{*} \mathcal{F}_{12}\right)(\Delta \otimes 1)\left(\mathcal{F}^{*} \mathcal{F}\right)$. Looking at the definition (2.11), this means that the left-hand side of (7.1) is the gauge transformation of $\phi$ under a gauge transformation $\mathcal{F} \rightarrow \mathcal{F}^{\prime}:=\mathcal{F} \sqrt{T}^{-1}$, which makes $\mathcal{F}$ unitary. Therefore the coassociator is unchanged under this gauge transformation, hence it remains minimal.

Proof of Lemma 3.2: We simply calculate

$$
\begin{aligned}
a_{1}^{\dagger} \star\left(a_{2} \star a_{3}\right) & =\left(a_{1}^{\dagger} \star a_{2}\right) \star a_{3} \tilde{\phi}_{123}^{-1} \\
& =\left(g_{12}+a_{2} \star a_{1}^{\dagger} \tilde{\mathcal{R}}_{12}\right) \star a_{3} \tilde{\phi}_{123}^{-1} \\
& =g_{12} a_{3} \tilde{\phi}_{123}^{-1}+a_{2} \star\left(a_{1}^{\dagger} a_{3}\right) \tilde{\phi}_{213} \tilde{\mathcal{R}}_{12} \tilde{\phi}_{123}^{-1} \\
& =g_{12} a_{3} \tilde{\phi}_{123}^{-1}+a_{2} \star\left(g_{13}+a_{3} \star a_{1}^{\dagger} \tilde{\mathcal{R}}_{13}\right) \tilde{\phi}_{213} \tilde{\mathcal{R}}_{12} \tilde{\phi}_{123}^{-1} \\
& =g_{12} a_{3} \tilde{\phi}_{123}^{-1}+a_{2} g_{13} \tilde{\phi}_{213} \tilde{\mathcal{R}}_{12} \tilde{\phi}_{123}^{-1}+\left(a_{2} \star a_{3}\right) \star a_{1}^{\dagger} \tilde{\phi}_{231}^{-1} \tilde{\mathcal{R}}_{13} \tilde{\phi}_{213} \tilde{\mathcal{R}}_{12} \tilde{\phi}_{123}^{-1} \\
& =g_{12} a_{3} \tilde{\phi}_{123}^{-1}+a_{2} g_{31} \tilde{\phi}_{231} \tilde{\mathcal{R}}_{1,(23)}+\left(a_{2} \star a_{3}\right) \star a_{1}^{\dagger} \tilde{\mathcal{R}}_{1,(23)},
\end{aligned}
$$

where (3.14) and $g_{31} \tilde{\mathcal{R}}_{13}=g_{13}$ was used in the last step. Now the first identity (3.23) follows immediately along these lines, omitting the inhomogeneous terms. To see the last one (3.25), observe that

$$
g_{12} \tilde{\phi}_{123}^{-1}=\left(g_{c}\right)_{12} \mathcal{F}_{1,(23)}^{-1} \mathcal{F}_{23}^{-1}
$$


because $g_{12} \mathcal{F}_{(12), 3}=g_{12}$, and similarly

$$
g_{31} \tilde{\phi}_{231} \tilde{\mathcal{R}}_{1,(23)}=\left(g_{c}\right)_{13} \mathcal{F}_{1,(23)}^{-1} \mathcal{F}_{23}^{-1}
$$

This implies that

$$
\left(g_{12} a_{3} \tilde{\phi}_{123}^{-1}+a_{2} g_{31} \tilde{\phi}_{231} \tilde{\mathcal{R}}_{1,(23)}\right) P_{23}^{-}=\left(\left(g_{c}\right)_{12} a_{3}+\left(g_{c}\right)_{13} a_{2}\right)\left(1-\delta_{32}^{23}\right) \mathcal{F}_{1,(23)}^{-1} \mathcal{F}_{23}^{-1}=0
$$

where we used the fact that the undeformed coproduct is symmetric. The second (3.24) follows as above using

$$
g_{31} \tilde{\phi}_{231} \tilde{\mathcal{R}}_{1,(23)} g^{23}=\delta_{1}^{2}
$$

or simply from (3.21).

Proof of Proposition 4.1: Relation (4.11) follows easily from

$$
\pi_{s}^{j}(u)\left(g_{c}\right)^{r s}=\pi_{l}^{r}(S u)\left(g_{c}\right)^{l j}
$$

To prove (4.10), consider

$$
\begin{aligned}
g^{i j} \hat{a}_{i} \hat{a}_{j} & =g^{i j} \mathcal{F}_{1}^{-1} \triangleright a_{i}\left(\mathcal{F}_{2,1}^{-1} \mathcal{F}_{a}^{-1}\right) \triangleright a_{j} \mathcal{F}_{2,2}^{-1} \mathcal{F}_{b}^{-1} \\
& =a_{k} a_{l} g^{i j} \pi_{i}^{k}\left(\mathcal{F}_{1}^{-1}\right) \pi_{j}^{l}\left(\mathcal{F}_{2,1}^{-1} \mathcal{F}_{a}^{-1}\right) \mathcal{F}_{2,2}^{-1} \mathcal{F}_{b}^{-1} \\
& =a_{k} a_{l} \pi_{n}^{j}\left(\gamma^{\prime}\right)\left(g_{c}\right)^{i n} \pi_{i}^{k}\left(\mathcal{F}_{1}^{-1}\right) \pi_{j}^{l}\left(\mathcal{F}_{2,1}^{-1} \mathcal{F}_{a}^{-1}\right) \mathcal{F}_{2,2}^{-1} \mathcal{F}_{b}^{-1}
\end{aligned}
$$

Now we use $\pi_{i}^{k}\left(\mathcal{F}_{1}^{-1}\right)\left(g_{c}\right)^{i n}=\left(g_{c}\right)^{k r} \pi_{r}^{n}\left(S \mathcal{F}_{1}^{-1}\right)$, therefore

$$
\begin{aligned}
g^{i j} \hat{a}_{i} \hat{a}_{j} & =a_{k} a_{l}\left(g_{c}\right)^{k r} \pi_{r}^{l}\left(\mathcal{F}_{2,1}^{-1} \mathcal{F}_{a}^{-1} \gamma^{\prime} S \mathcal{F}_{1}^{-1}\right) \mathcal{F}_{2,2}^{-1} \mathcal{F}_{b}^{-1} \\
& =a_{k} a_{l}\left(g_{c}\right)^{k l}
\end{aligned}
$$

because of (2.18).

Proof of Proposition 4.2: (4.13) follows easily from (4.10):

$$
\begin{aligned}
\hat{\partial}_{i}\left(g^{j k} \hat{a}_{j} \hat{a}_{k}\right) & =\hat{\partial}_{i}\left(\left(g_{c}\right)^{j k} a_{j} a_{k}\right) \\
& =\partial_{n} \pi_{i}^{n}\left(\mathcal{F}_{1}^{-1}\right) \mathcal{F}_{2}^{-1}\left(\left(g_{c}\right)^{j k} a_{j} a_{k}\right) \\
& =\partial_{n} \pi_{i}^{n}\left(\mathcal{F}_{1}^{-1}\right)\left(\left(g_{c}\right)^{j k} a_{j} a_{k}\right) \mathcal{F}_{2}^{-1} \\
& =2 a_{n} \pi_{i}^{n}\left(\mathcal{F}_{1}^{-1}\right) \mathcal{F}_{2}^{-1}+\left(\left(g_{c}\right)^{j k} a_{j} a_{k}\right) \partial_{n} \pi_{i}^{n}\left(\mathcal{F}_{1}^{-1}\right) \mathcal{F}_{2}^{-1} \\
& =2 \hat{a}_{i}+\left(g^{j k} \hat{a}_{j} \hat{a}_{k}\right) \hat{\partial}_{i},
\end{aligned}
$$


as claimed. Next, consider

$$
\begin{aligned}
\hat{\partial}_{i} \hat{a}_{j} & =\partial_{n} \pi_{i}^{n}\left(\mathcal{F}_{1}^{-1}\right) a_{l} \pi_{j}^{l}\left(\mathcal{F}_{2,1}^{-1} \mathcal{F}_{a}^{-1}\right) \mathcal{F}_{2,2}^{-1} \mathcal{F}_{b}^{-1} \\
& =\left(g_{c}\right)_{n l} \pi_{i}^{n}\left(\mathcal{F}_{1}^{-1}\right) \pi_{j}^{l}\left(\mathcal{F}_{2,1}^{-1} \mathcal{F}_{a}^{-1}\right) \mathcal{F}_{2,2}^{-1} \mathcal{F}_{b}^{-1}+a_{l} \pi_{j}^{l}\left(\mathcal{F}_{2,1}^{-1} \mathcal{F}_{a}^{-1}\right) \partial_{n} \pi_{i}^{n}\left(\mathcal{F}_{1}^{-1}\right) \mathcal{F}_{2,2}^{-1} \mathcal{F}_{b}^{-1} .
\end{aligned}
$$

The second term becomes $\hat{a}_{k} \hat{\partial}_{l} \mathfrak{R}_{i j}^{l k}$ as in (4.7), and the first is

$$
\begin{aligned}
\left(g_{c}\right)_{n l} \pi_{i}^{n}\left(\mathcal{F}_{1}^{-1}\right) \pi_{j}^{l}\left(\mathcal{F}_{2,1}^{-1} \mathcal{F}_{a}^{-1}\right) \mathcal{F}_{2,2}^{-1} \mathcal{F}_{b}^{-1} & =\pi_{l}^{t}\left(S \mathcal{F}_{1}^{-1}\right)\left(g_{c}\right)_{t i} \pi_{j}^{l}\left(\mathcal{F}_{2,1}^{-1} \mathcal{F}_{a}^{-1}\right) \mathcal{F}_{2,2}^{-1} \mathcal{F}_{b}^{-1} \\
& =\left(g_{c}\right)_{t i} \pi_{j}^{t}\left(S \mathcal{F}_{1}^{-1} \mathcal{F}_{2,1}^{-1} \mathcal{F}_{a}^{-1}\right) \mathcal{F}_{2,2}^{-1} \mathcal{F}_{b}^{-1} \\
& =\left(g_{c}\right)_{t i} \pi_{j}^{t}(\gamma)=\left(g_{c}\right)_{t i} \pi_{l}^{t}\left(S \mathcal{F}_{1}^{-1}\right) \pi_{j}^{l}\left(\mathcal{F}_{2}^{-1}\right) \\
& =\left(g_{c}\right)_{t l} \pi_{i}^{t}\left(\mathcal{F}_{1}^{-1}\right) \pi_{j}^{l}\left(\mathcal{F}_{2}^{-1}\right)=g_{i j}
\end{aligned}
$$

using (2.17).

Proof of Proposition 4.3: Since $\pi$ is a unitary representation, we have

$$
\begin{aligned}
\hat{a}_{i}^{*} & =\mathcal{F}_{2} a_{j}^{*} \pi_{j}^{i}\left(\mathcal{F}_{1}\right)=\mathcal{F}_{2} a_{k}\left(g_{c}\right)^{k j} \pi_{j}^{i}\left(\mathcal{F}_{1}\right) \\
& =\mathcal{F}_{2} a_{k}\left(g_{c}\right)^{n i} \pi_{n}^{k}\left(S \mathcal{F}_{1}\right) \\
& =a_{l} \pi_{k}^{l}\left(\mathcal{F}_{2,1}\right)\left(g_{c}\right)^{i n} \pi_{n}^{k}\left(S \mathcal{F}_{1}\right) \mathcal{F}_{2,2} \\
& =a_{l} \pi_{k}^{l}\left(\mathcal{F}_{2,1} S \mathcal{F}_{1}\right) \mathcal{F}_{2,2} g^{i t} \pi_{t}^{k}\left(\gamma^{\prime-1}\right) \\
& =a_{l} \pi_{t}^{l}\left(\mathcal{F}_{2,1} S \mathcal{F}_{1} \gamma^{\prime-1}\right) \mathcal{F}_{2,2} g^{i t} \\
& =a_{l} \pi_{t}^{l}\left(\mathcal{F}_{1}^{-1}\right) \mathcal{F}_{2}^{-1} g^{i t} \\
& =\hat{a}_{t} g^{i t}
\end{aligned}
$$

where (2.16) was essential.

\section{References}

[1] A. Yu. Alekseev, A. Recknagel, V. Schomerus, "Non-commutative World-volume Geometries: Branes on SU(2) and Fuzzy Spheres", JHEP 9909, 023 (1999).

[2] A. Yu. Alekseev, A. Recknagel, V. Schomerus, "Brane Dynamics in Background Fluxes and Non-commutative Geometry", hep-th/0003187.

[3] B. L. Cerchiai, J. Wess, "q-Deformed Minkowski Space based on a q-Lorentz Algebra", Eur.Phys.J. C5 (1998) 553-566; M. Fichtmueller, A. Lorek, J. Wess, "q-deformed Phase Space and its Lattice Structure", Z.Phys. C71 (1996) 533-538. 
[4] M. Chaichian, A. Demichev, P. Presnajder, "Quantum Field Theory on the Noncommutative Plane with $E_{q}(2)$ Symmetry", J.Math.Phys. 41 (2000) 1647-1671.

[5] T. L. Curtright and C. K. Zachos, Phys. Lett. B243 (1990), 237. T. L. Curtright, G. I. Ghandour, C. K. Zachos, J. Math. Phys. 32 (1991), 676.

[6] V. Drinfel'd, "Quantum Groups" Proceedings of the International Congress of Mathematicians, Berkeley, 1986 A.M. Gleason (ed.), p. 798, AMS, Providence, RI.

[7] V. G. Drinfel'd, "On Quasitriangular Quasi-Hopf algebras and a group closely connected with $\operatorname{Gal}(\bar{Q} / Q)$ ", Leningrad Math. J. 2, No.4, 829 (1991); V. G. Drinfel'd, "Quasi-Hopf Algebras", Leningrad Math. J. 1, No.6, 1419 (1991).

[8] L.D.Faddeev, N.Yu.Reshetikhin, L.A.Takhtajan. "Quantization of Lie Groups and Lie Algebras" Algebra Anal. 1178 (1989).

[9] G. Fiore, "The $S O_{q}(N)$ - Symmetric Harmonic Oscillator on the Quantum Euclidean Space $R_{q}^{N}$ and its Hilbert Space Structure" Int. J. Mod. Phys A8, 4679 (1993).

[10] G. Fiore, "Drinfel'd Twist and $q$-Deforming Maps for Lie Group Covariant Heisenberg Algebras", Rev.Math.Phys. 12 (2000) 327-359; q-alg/9708017.

[11] G. Fiore, P. Schupp, "Identical Particles and Quantum Symmetries", Nucl.Phys. B470 (1996) 211-235; hep-th/9508047.

[12] H. Grosse, J. Madore, H. Steinacker, "Field Theory on the $q$-deformed Fuzzy Sphere I", hep-th/0005273, to appear in J. Geom. Phys.

[13] H. Grosse, C. Klimcik, P. Presnajder, "Towards Finite Quantum Field Theory in NonCommutative Geometry", Int. J. Theor. Phys. 35, 231 (1996), hep-th/9505175.

[14] A. Hebecker and W. Weich, "Free Particle in $q$ - deformed Configuration space". Lett. Math. Phys. 26, 245 (1992).

[15] A.P.Isaev, Z.Popowicz, "Quantum Group Gauge Theories and Covariant Quantum Algebras", Phys.Lett. B307 (1993) 353-361.

[16] B. Jurco, "More on quantum groups from the the quantization point of view", Commun.Math.Phys. 166 (1994) 63-78; hep-th/9301092.

[17] T. Kohno, Ann. Inst. Fourier (Grenoble) 37 (1987), 139; Adv. Stud. in Pure. Math. 1 (1987), 189; Sugaku 41 (1989), 305; and references therein. 
[18] G. Mack, V. Schomerus, "Quasi Hopf quantum symmetry in quantum theory" Nucl. Phys. B 370, 185 (1992).

[19] J. Madore, "The Fuzzy Sphere", Class. Quant. Grav. 9, 69 (1992).

[20] S. Majid, "Introduction to Braided Geometry and q-Minkowski Space", hep-th/9410241.

[21] S. Majid, "Foundations of Quantum Groups", Cambridge University Press, 1995.

[22] U. Meyer, "Wave Equations on q-Minkowski space", Commun.Math.Phys. 174 (1995) 457-476.

[23] R. Oeckl, "Braided Quantum Field Theory", Commun. Math. Phys. 217 (2001) 451-473.

[24] P. Podleś, "Quantum Spheres". Lett. Math. Phys. 14, 193 (1987).

[25] P. Podleś, "Solutions of Klein-Gordon and Dirac equations on quantum Minkowski spaces", Commun.Math.Phys. 181, (1996) 569-586

[26] N. Seiberg, E. Witten, "String Theory and Noncommutative Geometry", JHEP 9909 (1999) 032.

[27] H. Steinacker, "Quantum Anti-de Sitter space and sphere at roots of unity", Adv. Theor. Math. Phys. 4, Nr. 1 (2000); hep-th/9910037. 